\title{
Constrained observability method in static structural system identification
}

\begin{tabular}{|r|l|}
\hline Journal: & Structural Control and Health Monitoring \\
\hline Manuscript ID & STC-16-0194.R2 \\
\hline Wiley - Manuscript type: & Research Article \\
\hline Complete List of Authors: & $\begin{array}{l}\text { Lei, Jun; Tongji University, Department of Bridge Engineering } \\
\text { Nogal, Marí; University of Dublin Trinity College, Department of Civil, } \\
\text { Structural \& Environmental Engineering } \\
\text { Lozano Galant, Jose Antonio; University of Castilla-La Mancha, Department } \\
\text { of Civil Engineering } \\
\text { Xu, Dong; Tongji University, Deparment of Bridge Engineering } \\
\text { Turmo, Jóse; Universitat Politecnica de Catalunya, Department of Civil and } \\
\text { Environmental Engineering }\end{array}$ \\
\hline Keywords: & $\begin{array}{l}\text { structural system identification, stiffness method, observability method, } \\
\text { nonlinear constraint, essential set, static }\end{array}$ \\
\hline
\end{tabular}

\section{SCHOLARONE ${ }^{m}$}

Manuscripts 
1

2

3

4

5

6

7

8

9

10

11

12

13

14

15

16

17

18

19

20

21

22

23

24

25

26

27

28

29

30

31

32

33

34

35

36

37

38

39

40

41

42

43

44

45

46

47

48

49

50

51

52

53

54

55

56

57

58

59

60

1 Journal: Structural Control and Health Monitoring

2 Title: Constrained observability method in static structural system

3 identification

4

5 Authors:

6 Jun Lei, María Nogal, Jose Antonio Lozano-Galant, Dong Xu( Corresponding Author),

7 Jose Turmo

8

9 Corresponding author:

10 Dong $\mathrm{Xu}$,

11 Prof., Ph.D.

12 Department of Bridge Engineering at Tongji University

13 1239, Siping Road, Shanghai, China

14 Post: 200092

15 Email: xu_dong@tongji.edu.cn

16 Tel: 021-65980953

17 Fax: 021-65980953

18

19

20

21

22

23

24

25

26

27

28

29

30

31

32

33

34

35

36

37

38 


\title{
Constrained observability method in static structural system identification
}

\begin{abstract}
Summary
Identifiability of parameters in structural system identification (SSI) is of primary importance in any SSI method. It depends on the number and the location of the measurements, which is directly linked with sensor configuration. In this paper, under the framework of SSI by observability method (OM), the number of necessary measurements to identify all parameters of structural system was clarified first. Then an example was solved step by step to show the lacking constraints among unknowns in SSI by OM. In a frame example, it was found that no measurement set having as many measurements as the number of unknowns was able to identify all parameters. To further understand this phenomenon, the observability of a simply supported beam was analyzed in an exhaustive way with respect to 252 possible measurement sets. It turned out that three quarters of these sets were not able to identify all the parameters. In order to solve this issue, for the very first time, SSI by constrained observability method (COM), which appends the nonlinear constraints to SSI by OM, was proposed. With SSI by COM applied, the observability of structural parameters with respect to the 252 sets was greatly improved. Finally, the efficacy of this method was verified by a 13 storey frame building.
\end{abstract}

Keyword: structural system identification; stiffness method; observability method; nonlinear constraint; essential set; static

\section{Section 1: Introduction}

Structural System Identification (SSI) has long been an intriguing topic in the field of civil engineering. SSI can be conceptualized as the process of simulating the structural behavior by mathematical models. Based on the type of the excitation, methods for SSI can be categorized as static ${ }^{[1-8]}$ or dynamic ${ }^{[9-12]}$. The dynamic SSI requires the use of mass, stiffness and damping properties while the static method only requires the use of stiffness properties ${ }^{[13]}$. It was pointed out by Sanaye ${ }^{[14]}$ that static SSI might be more interesting than dynamic SSI in cases when only the estimation of stiffnesses is targeted. In many cases, the estimation of stiffness is sufficient for condition assessment of the structure. In the non-destructive test of bridges, slow moving load can be applied as a quasi-static load ${ }^{[15-18]}$. In curvature-based methods ${ }^{[15,18]}$, the curvatures of the displacement influence lines of damaged and undamaged beams under moving load are obtained first and the damages are located by the irregular variation in curvatures. Boumechra ${ }^{[16]}$ approximated the inverse of the global stiffness matrix by a Neumann series. SSI is posed as the problem of finding the correction coefficients of the stiffness of each element that minimize the difference between the measured response and the predicted response under moving load. This method can accurately localize and quantify the damage with the displacements of selected nodes. Providing that the structure behaves linearly, Maxwell law of reciprocal deflection can be applied to limit the number of sensors installed in the structure subjected to this type of load, which might make the method more attractive ${ }^{[17]}$. This strategy was also adopted by other researchers to achieve dense measurements without using many sensors ${ }^{[18,19]}$. The elastic damage load theorem is derived by $\mathrm{Choi}^{[19]}$ for statically determinate beam. The location of 
damage in statically determinate beams is reflected by the variation in the shape of deflection.

However, this method is not able to quantify the extent of damage and its application is limited to statically determinate structures. From the point of the interpretability of the model, SSI methods can be classified as physics-based models (e.g. finite element models (FEM)) or nonphysics based models (the neural networks models ${ }^{[20-22]}$, autoregressive models ${ }^{[23-25]}$ or rational polynomial models ${ }^{[26-28]}$ ). Physics-based models and non-physics based models are respectively, called parametric models and non-parametric models. However, in effect, both types of models have parameters. In the physics-based models, the parameters represent the structural characteristics, which might be elastic modulus, inertias, areas, mass or damping ratios. Conversely, the parameters of the non-parametric models play the role of weight factors of the adopted basis functions. These parameters have no physical meaning and are determined by minimizing the discrepancy between the predicted structural response and the real-life structural response. From a statistical perspective, SSI methods can be categorized as probabilistic methods ${ }^{[8,12,29-32]}$ or deterministic methods ${ }^{[13,33,34]}$. In probabilistic SSI, each structural parameter is treated as random variable and is assumed to follow a prescribed distribution using prior information. A set of parameters will be formed by sampling each distribution once. A set of parameters represents a possible model for the structure. All these models are designated as trial models ${ }^{[8,12,29,30]}$. Many trial models will be generated and be evaluated by the discrepancy between the response (e.g. strains, deflections or frequencies) calculated from them and the measured one in real-life. A specified proportion of the generated models which have the lowest discrepancy will be retained as the probable models. The mean and standard deviation of the assumed distribution of each structural parameter, will be updated by the statistical inference from the values of that parameter in all these probable models. These updated distributions will be used to generate new trial models and restart the process until the discrepancy between the response calculated from the estimated parameters and the measured response reaches a specified threshold. The strengths of probabilistic methods are that they not only provide the estimation of the parameters but also a measure to assess the confidence in the estimates. However, the computation cost of the probabilistic methods increases exponentially with the number of parameters due to the combinatorial consideration linked with the generation of parameter set. In contrast to the probabilistic method, the deterministic methods try to pinpoint a best model yielding the closest response to the one measured. The main drawback of these methods is that evaluation of the confidence in the estimates is not available in the theoretical formulation. However, this might be overcome by the post analysis of the estimates obtained by using different sets of data generated by Monte-Carlo method.

A vital issue for all the methods mentioned above is whether the parameters within the structural system can be identified or not. In practice, large amount of data is required to train the non-parametric models, i.e. to obtain the parameters. In contrast, fewer measurements are required by the parametric models since the mathematical relationships, i.e. the system of equations, are well defined in these models. However, in the context of the parametric methods, one might ask: Are the parameters within the structure identifiable given a particular measurement set? A generalized interpretation of this question is: Is the available information sufficient to specify a unique solution of a system of equations? In fact, this issue is addressed by the Observability Method $(\mathrm{OM})^{[35,36]}$. This mathematical tool provides the information whether all the unknowns or a subset of the unknowns can be uniquely determined or not. Even though OM was originally conceived to deal with linear system of equations, it have been also applied to inequalities ${ }^{[35]}$ and to nonlinear systems ${ }^{[36]}$. It has been applied extensively to 
parameter estimation in different engineering fields, including water transport network ${ }^{[37-39]}$, 128 traffic network $^{[40-43]}$ or power systems ${ }^{[44-46]}$.

OM was first introduced into the static SSI by Lozano-Galant ${ }^{[33]}$. This is a deterministic static the tailor-made implementation of OM in static SSI, the required system of equations is derived by algebraic operations on the nodal equilibrium equations obtained from direct stiffness method, which makes SSI by OM a physics-based method, and any subset of the solution is regarded as observable if the corresponding rows in the null space of the acquired coefficient matrix are composed of only zeros. The efficacy of this method was verified by its application in the identification of trusses, beams, frame structures ${ }^{[33]}$. Also, this innovative method can be applied in cable-stayed bridges ${ }^{[47]}$, wherein the structural audacity and lightness makes these structures sensitive to dynamic and static load cases in both service and construction stage ${ }^{[48,49]}$. It can also help the decision making during the maintenance of structures ${ }^{[50]}$. A peculiarity of this method is that the identification of parameters is carried out in a recursive manner. That is, the consecutive identifications rely on the existing information in the measurements and/or the identification results from the preceding steps. However, in this method, the parameters to be identified should be activated by the external load. Or in other words, the displacements should be sensitive to the parameters under the given load. For instance, in the case of simply supported beams subjected to vertical loads, the vertical deflections have nothing to do with the axial stiffnesses. Hence, it is impossible to identify the axial stiffnesses of the simply supported beams by just measuring these vertical deflections in this case.

As mentioned above, the identifiability of the structure by both, parametric method and nonparametric method relies on the available information. This is due to the fact that the information carried by the measurements bridges the mathematical models with the real-life structure. From the point of identifiability, more sensors, i.e. more information, are generally desirable. On the converse, using less sensors means reducing the chance of measuring and processing redundant measurements. In any SSI method, different measurement sets might be used for parameter estimation. However, with regard to the selection of measurements, one might be faced with the limit induced by the extended dimension of structures, the operational costs, the accessibility. A more practical question is posed as how to find the optimal number of measurements and the location of the sensors for successful identifications. A similar issue was addressed in the parameter estimation of power system under $\mathrm{OM}^{[46]}$. In this problem, the system can be specified by $n$ parameters and $m$ potential measurements $(m>n)$. It is preferable to choose $n$ out of $m$ measurements to identify all the $n$ parameters of the system. If a measurement set of $n$ measurements is able to identify all the $n$ parameters, and the drop of any measurement in this set fails to do so, then this set is defined as the essential set. Generally, such a set is not unique. OM is introduced into the aforementioned disciplines to serve two purposes: (1) In the preliminary stage of designing the measurement configuration, provide a solution of essential set to ensure that all the parameters of the system can be identified; (2) In the operation stage of the monitoring system, if any of the measuring devices is out of service, determine the observability of the system parameters. In the context of SSI, it was pointed out that if the number of measurements was less than the number of unknown parameters, the system would become indeterminate ${ }^{[13]}$. This is to say, in this case, only a subset of the parameters might be identified, which is referred as partial observability in this paper. Sanayei ${ }^{[51]}$ also concluded that the number of measurement must be greater than or equal to the number of parameters, as a necessary condition for the solution to exist. Moreover, even if the number of measurements equals to the number of unknowns, if those are not properly chosen, the system might still be indeterminate. An increase of the number of measurements or a better placement of the sensors 
can lead to an increase in the number of the identified parameters, which will be referred as increase of observability in this paper. Eventually, this process will lead to the identification of all parameters and to make the system determinate, which is referred as full observability.

A wide range of proposals and methods dealing with the placement of sensors in SSI can be found. Sanayei ${ }^{[52]}$ used a heuristic method to seek near-optimal placement of sensors for structures under non-destructive test. It was pointed out that the structure was identifiable on the premise that the number of measurements was more than the number of unknown parameters. Cha ${ }^{[53]}$ proposed a multi-objective genetic algorithm for optimal placement of control devices and sensors in seismically excited civil structures. A series of optimal sensor placement techniques for monitoring vibration response, including effective influence method, the driving point residue kinetic energy and modified variance method, are discussed by Chang ${ }^{[54]}$. Jin ${ }^{[55]}$ applied an improved harmony search optimization algorithm and the modal assurance criterion to find the optimal sensor placement for the identification of the mode parameters. Malings ${ }^{[56]}$ proposed the sensor placement as an optimization problem with respect to the conditional entropy and the value of information.

It is pointed out that the majority of the existing literature on measurement selection focuses on dynamic SSI. In static SSI by OM, the identifiability of the structural parameters also relies on a proper measurement selection. To deal with this issue, the observability trees method was proposed by Lozano-Galant ${ }^{[2]}$. The order of identification in the sequence of identified parameters with any given measurement set can be analyzed graphically by the observability tree technique. A forward and backward strategy was adopted then to find the essential sets, i.e. to select those measurements able to maintain the observability flow and thereby to identify the desired structural parameters. Note that the notion of essential set in power systems and the notion of essential set in SSI by OM are used interchangeably in this paper. It was found that improper selection of the measurement set cuts off the observability flow and thus leads to fail to identify all the unknowns even with some measurement sets which have more measurements than the essential sets. However, the underlying reason for this deficiency is not clear and the pertinent solution remained to be found at that time. For the aforementioned reasons, the aim of this paper is twofold: (1) to clarify the underlying reason why some measurement sets expected to be able to identify all structural parameters turn out to be unqualified to do so in SSI by OM and (2) to provide a method to alleviate or solve this problem.

The rest of this paper is organized as follows. In section 2, the essential set is conceptualized first and two simple examples are used to illustrate the deficiency of SSI by OM. And then the observability of the structural parameters in a simply supported beam is analyzed in an exhaustive way for 252 enumerated measurement sets. Based on the analysis of the result, two reasons of the unqualification of some measurement sets to be essential sets are revealed. In the following section, the constrained observability method (COM) is proposed as the solution to this deficiency. The effectiveness and robustness of COM is justified by the improvement of observability on the two examples proposed in Section 2. Next, in section 4, a 13-storey frame with a large number of Degrees Of Freedom (DOFs) is studied to further illustrate the strength of this COM. Finally, some conclusions are drawn in section 5.

\section{Section 2: Inadequacy of OM}

217 Concerning beam element in 2D structural models, the nodal equilibrium equations from direct 218 stiffness method can be expressed as: 
$K \cdot \delta=\left[\sum_{j=1}^{N_{e}} K_{e}\left(E_{j} A_{j}, E_{j} I_{j}\right)\right] \cdot\left\{\begin{array}{c}u_{1} \\ v_{1} \\ w_{1} \\ \vdots \\ u_{N_{n}} \\ v_{N_{n}} \\ w_{N_{n}}\end{array}\right\}=\left\{\begin{array}{c}H_{1} \\ V_{1} \\ M_{1} \\ \vdots \\ H_{N_{n}} \\ V_{N_{n}} \\ M_{N_{n}}\end{array}\right\}=f$,

219

220

221

222

223

224

225

226

227

228

229

230

231

232

233

234

235

236

237

238

239

240

241

242

243

244

245

246

247

248

249

250

251

252

253

254

where $N_{e}$ and $N_{n}$, respectively indicate the number of elements and the number of nodes in the FEM; $u_{i}, v_{i}$ and $w_{i}$, respectively, indicate the horizontal, vertical and rotational displacements of the node $\mathrm{i} ; H_{i}, V_{i}$ and $M_{i}$, respectively, indicate the external horizontal forces, vertical forces and moments applied on node $i$. The mechanical parameters for element $j$ include elastic modulus, $E_{j}$, areas, $A_{j}$, and inertias, $I_{j}$.

In either static or dynamic SSI, the target parameters to be identified are axial stiffnesses and flexural stiffnesses. In SSI by OM, when the axial stiffness, $E_{j} A_{j}$, appears in the equation, it is regarded as one unknown instead of being regarded as a product of the unknown modulus, $E_{j}$, and the unknown area, $A_{j}$. This principle goes the same in the case of flexural stiffness. For simplicity, the axial stiffness and the flexural stiffness will be, respectively, denoted as $E A_{j}$ and $E I_{j}$ in SSI by OM. In addition, truss elements are treated as a degenerate case of beam elements and their flexural stiffnesses, $E I_{j}$, is set as null.

In Eq. (1), the symbol $\Sigma$ represents the operation of assembling all element stiffness matrices into the global stiffness matrix K. In fact, the information of the geometry, or the element connectivity, is introduced into the equations by this operation. Once the geometry of the structure, the boundary conditions and the load case are specified, then the nodal displacements, $\delta$, will be uniquely determined by any particular set of axial stiffnesses, $E A_{j}$, and flexural stiffnesses, $E I_{j}$. In the case of there being $N_{A}$ unknown axial stiffnesses and $N_{F}$ unknown flexural stiffnesses, this is saying that these $N_{A}+N_{F}$ mechanical parameters uniquely specify a set of displacements of the structure. Conversely, in the inverse analysis of this problem, due to the linearity of this system, it is expected that $N_{A}+N_{F}$ measurements of the displacements will suffice to identify all the unknown parameters. For simplicity, the ability to identify all the parameters of the structure is referred as full observability in this paper. Meanwhile, if merely a subset of these parameters are identifiable, then it is referred as partial observability. Hence, in the essential sets (the sets containing the necessary and sufficient number of measurements to achieve full observability), the number of required measurements is $N_{A}+N_{F}$. However, this statement has been verified elsewhere ${ }^{[2]}$ and it was found that $N_{A}+N_{F}$ measurements led to full observability in SSI by OM under certain conditions. This is to say, even with $N_{A}+N_{F}$ measurements, full observability might not be achieved due to improper selection of the measurement set, which is related with the placement and type of measuring devices. An explanation provided formerly was that the recursive steps stopped too early without identifying all parameters. However, the underlying cause for this premature end of the recursive steps was not uncovered at that time. This is one of the major interests of this paper, which can also be employed for the sensor placement strategy.

Eq. (1) can be rearranged for practical purposes and the system of equations to be solved by OM can be transformed into: 
$B \cdot z=D$,

255

256

257

258

259

260

261

262

263

264

265

266

267

268

269

270

271

272

273

274

275

276

277

278

279

280

281

282

283

284

285

286

287

288

289

290

291

292

293

294

295

296

where $\mathrm{B}$ and $\mathrm{D}$ are a matrix and a vector whose entries are all known, respectively, and $\mathrm{z}$ is the vector of unknowns. This is the result of applying static condensation technique together with the separation of each column, with regard to different unknowns, into several columns and the merger (addition) of those resultant columns related with the same unknowns in the equilibrium equations (Eq. (1)), derived from the direct stiffness method. After these algebraic operations, the information of measurements, is absorbed in the coefficient matrix $B$ and in the vector $D$. Apart from the information of measurements, the vector $D$ contains the information of both the external loads and the boundary conditions, which are all assumed to be known. The unknowns $z$ can be always of two types: (1) monomials of degree one, e.g. $\left\{E A_{j}, E I_{j}, u_{i}, v_{i}, w_{i}, H_{i}, V_{i}, M_{i}\right\}$, or (2) monomials of degree two, e.g. $\left\{E I_{j} u_{i}, E I_{j} v_{i}, E I_{j} w_{i}, E A_{j} u_{i}, E A_{j} v_{i}\right\}$. Note that both, $E A_{j}$ and $E I_{j}$ are regarded as monomials of degree one. (For more technical details, readers are strongly recommended to review ${ }^{[3,57]}$ ). The occurrence of these components of the unknown $z$ is due to the fact that Eq. (2) is essentially established by nodal force equilibrium.

If the vertical deflection, $v_{i}$, or the rotation, $w_{i}$, appearing in these products, $E I_{j} v_{i}$ or $E I_{j} w_{i}$, are measured, then the flexural stiffness, $E I_{j}$, can be uncoupled and separated from those and the vertical deflection, $v_{i}$, or the rotation, $w_{i}$, will be absorbed in $B$. Otherwise, these products themselves will appear in $z$ in the form of monomials with degree of two and, as per requirements of $\mathrm{OM}$, be regarded as linear in $z$. This is to say, even though the physical unknowns for a given problem might be $E I_{j}$ and $w_{i}, \mathrm{z}$ may contain three different unknowns, $E I_{j}, w_{i}$ and $E I_{j} w_{i}$. Due to the limit of sensor investment, it is not likely to measure all displacements in the structure. As a consequence, these products, $E I_{j} v_{i}$ or $E I_{j} w_{i}$, and the flexural stiffness, $E I_{j}$, which is obtained by the uncoupling of these products, both appear in the unknowns $z$. Likewise, the simultaneous occurrence of $E A_{j} u_{i}$ and $E A_{j}$ is ascribed to the nodal equilibrium of axial forces. Furthermore, it is worth mentioning that in frame structures, due to the coupling of the axial displacements and the vertical deflections from different members, the product of axial stiffness and vertical deflection, $E A_{j} v_{i}$, and the product of flexural stiffness and axial displacement, $E I_{j} u_{i}$, also appear in the unknowns z.

From a mathematical point, it is straightforward that if the unknowns of a system are coupled, then these coupled unknowns should satisfy certain constraints. Nevertheless, this requirement is not satisfied in SSI by OM and therefore sometimes leads to the failure to identify all parameters, or in other words, to achieve full observability.

In order to clarify this deficiency induced by the lack of constraints, SSI by OM on a 4-node simply supported beam depicted in Figure 1, is solved step by step. Meanwhile, this analysis also sheds light on the peculiarity of this method. For simplicity, it is assumed that the flexural stiffnesses of elements 1 and $3, E I_{1}$ and $E I_{3}$, the length of element, $\mathrm{L}$, and the external vertical load at node $2, V_{2}$, are known. Since the axial stiffness is not activated by this load case, the areas of the elements are not considered, i.e. $N_{A}=0$. Correspondingly, the terms associated with axial behavior are removed from the general equation by OM. Thus, the target parameter is the flexural stiffness of the element 2 (in red in Figure 1), $I_{2}$, i.e. $N_{F}=1$. Then it is anticipated that one measurement suffices to achieve full observability since $N_{A}+N_{F}=1$. Assume that the vertical deflection of the node $2, v_{2}$, is measured.

In the first step, the system of equations given by $\mathrm{OM}$ is as follows: 
$B_{1} \cdot z_{1}=\left(\begin{array}{ccccccccccc}0 & 0 & 0 & 0 & 0 & 0 & 0 & \frac{4 E I_{1}}{L} & \frac{2 E I_{1}}{L} & 0 & 0 \\ \frac{12 v_{2}}{L^{3}} & -\frac{12}{L^{3}} & \frac{6}{L^{2}} & \frac{6}{L^{2}} & 0 & 0 & 0 & -\frac{6 E I_{1}}{L^{2}} & -\frac{6 E I_{1}}{L^{2}} & 0 & 0 \\ \frac{6 v_{2}}{L^{2}} & -\frac{6}{L^{2}} & \frac{4}{L} & \frac{2}{L} & 0 & 0 & 0 & \frac{2 E I_{1}}{L} & \frac{4 E I_{1}}{L} & 0 & 0 \\ -\frac{12 v_{2}}{L^{3}} & \frac{12}{L^{3}} & -\frac{6}{L^{2}} & -\frac{6}{L^{2}} & 0 & 0 & \frac{12 E I_{3}}{L^{3}} & 0 & 0 & \frac{6 E I_{3}}{L^{2}} & \frac{6 E I_{3}}{L^{2}} \\ \frac{6 v_{2}}{L^{2}} & -\frac{6}{L^{2}} & \frac{2}{L} & \frac{4}{L} & 0 & 0 & \frac{6 E I_{3}}{L^{2}} & 0 & 0 & \frac{4 E I_{3}}{L} & \frac{2 E I_{3}}{L} \\ 0 & 0 & 0 & 0 & 0 & 0 & \frac{6 E I_{3}}{L^{2}} & 0 & 0 & \frac{2 E I_{3}}{L} & \frac{4 E I_{3}}{L} \\ 0 & 0 & 0 & 0 & -1 & 0 & 0 & \frac{6 E I_{1}}{L^{2}} & \frac{6 E I_{1}}{L^{2}} & 0 & 0 \\ 0 & 0 & 0 & 0 & 0 & -1 & -\frac{12 E I_{3}}{L^{3}} & 0 & 0 & -\frac{6 E I_{3}}{L^{2}} & -\frac{6 E I_{3}}{L^{2}}\end{array}\right) \cdot\left(\begin{array}{c}E I_{2} \\ E I_{2} v_{3} \\ E I_{2} w_{2} \\ E I_{2} w_{3} \\ V_{1} \\ V_{4} \\ v_{3} \\ w_{1} \\ w_{2} \\ w_{3} \\ w_{4}\end{array}\right)=\left(\begin{array}{c}M_{1}+\frac{6 E I_{1} v_{2}}{L^{2}} \\ V_{2}-\frac{12 E I_{1} v_{2}}{L^{3}} \\ M_{2}+\frac{6 E I_{1} v_{2}}{L^{2}} \\ V_{3} \\ M_{3} \\ M_{4} \\ 0 \\ \frac{12 E I_{1} v_{2}}{L^{3}} \\ 0\end{array}\right)=D_{1}$

297

298

299

300

301

302

303

304

305

306

307

308

309

310

311

312

313

314

In Eq. (3), it is seen that the vertical deflection $v_{2}$, flexural stiffnesses $E I_{1}$ and $E I_{3}$ are absorbed in both the matrix $B_{1}$ and the vector $D_{1}$. The solution of this system can be expressed as the sum of a particular solution $\mathrm{z}_{\mathrm{p} 1}$ and any linear combination of the bases in the null space of $B_{1}$, as presented in Eq. (4). This particular solution as well as the bases of the vectorial space of any possible mathematical solution can be obtained by many commercial packages, e.g. Matlab ${ }^{[58]}$ and Maple ${ }^{[59]}$. For this structure, it can be seen that, in the null space of the matrix $B_{1}$, i.e. $V_{n 1}$, the element of the rows associated with $\left\{V_{1}, V_{4}, w_{1}, w_{4}\right\}$, given in bold, are all null. Hence the values of them are not affected by the coefficients $\rho_{1,1}, \rho_{1,2}$ and $\rho_{1,3}$. This is saying $\left\{V_{1}, V_{4}, w_{1}, w_{4}\right\}$ are constant, unique, known and, hence, observable. Another thing is that $z_{1}$ contains $\left\{E I_{2}, v_{3}, w_{2}, w_{3}, E I_{2} v_{3}, E I_{2} w_{2}, E I_{2} w_{3}\right\}$ and these unknowns should satisfy certain constraints, e.g. $\left\{E I_{2} \cdot v_{3}=E I_{2} v_{3}\right\}$. This gives rise to the nonlinearity of this problem. However, as linearity is assumed, these constraints are not considered in SSI by OM yet, which leads to the failure of the identification of $E I_{2}$.

$$
Z_{1}=z_{p 1}+V_{n 1} \cdot \boldsymbol{\rho}_{\mathbf{1}}=\left(\begin{array}{c}
E I_{2} \\
E I_{2} v_{3} \\
E I_{2} w_{2} \\
E I_{2} w_{3} \\
\boldsymbol{V}_{\mathbf{1}} \\
\boldsymbol{V}_{\mathbf{4}} \\
v_{3} \\
\boldsymbol{w}_{\mathbf{1}} \\
\boldsymbol{w}_{2} \\
w_{3} \\
w_{4}
\end{array}\right)_{\text {particular }} \quad+\left(\begin{array}{ccc}
\frac{1}{v_{2}} & -\frac{L}{v_{2}} & 0 \\
1 & 0 & 0 \\
0 & 1 & 0 \\
0 & 1 & 0 \\
\mathbf{0} & \mathbf{0} & \mathbf{0} \\
\mathbf{0} & \mathbf{0} & \mathbf{0} \\
0 & 0 & -L \\
\mathbf{0} & \mathbf{0} & \mathbf{0} \\
\mathbf{0} & \mathbf{0} & \mathbf{0} \\
0 & 0 & 1 \\
0 & 0 & 1
\end{array}\right) \cdot\left(\begin{array}{c}
\rho_{1,1} \\
\rho_{1,2} \\
\rho_{1,3}
\end{array}\right)
$$

In the next recursive step, the unknowns observed previously are incorporated into the input of SSI by OM. This is, the up-to-date measurement set for the current recursive step is $\left\{w_{1}, w_{2}\right.$ and $\left.v_{2}\right\}$ and the reactions, $V_{1}$ and $V_{4}$, are also regarded as known. Note that a renewed input will update Eq. (2) and thus new parameters might be observed. The updated system of equations obtained in the next recursive step is as follows:

$$
B_{2} \cdot z_{2}=\left(\begin{array}{cccccc}
0 & 0 & 0 & 0 & 0 & 0 \\
\frac{12 v_{2}}{L^{3}}+\frac{6 w_{2}}{L^{2}} & -\frac{12}{L^{3}} & \frac{6}{L^{2}} & 0 & 0 & 0 \\
\frac{6 v_{2}}{L^{2}}+\frac{4 w_{2}}{L} & -\frac{6}{L^{2}} & \frac{2}{L} & 0 & 0 & 0 \\
-\frac{12 v_{2}}{L^{3}}-\frac{6 w_{2}}{L^{2}} & \frac{12}{L^{3}} & -\frac{6}{L^{2}} & \frac{12 E I_{3}}{L^{3}} & \frac{6 E I_{3}}{L^{2}} & \frac{6 E I_{3}}{L^{2}} \\
\frac{6 v_{2}}{L^{2}}+\frac{2 w_{2}}{L} & -\frac{6}{L^{2}} & \frac{4}{L} & \frac{6 E I_{3}}{L^{2}} & \frac{4 E I_{3}}{L} & \frac{2 E I_{3}}{L} \\
0 & 0 & 0 & \frac{6 E I_{3}}{L^{2}} & \frac{2 E I_{3}}{L} & \frac{6 E I_{3}}{L} \\
0 & 0 & 0 & 0 & 0 & 0 \\
0 & 0 & 0 & -\frac{12 E I_{3}}{L^{3}} & -\frac{6 E I_{3}}{L^{2}} & -\frac{6 E I_{3}}{L^{2}}
\end{array}\right) \cdot\left(\begin{array}{c}
E I_{2} \\
E I_{2} v_{3} \\
E I_{2} w_{3} \\
v_{3} \\
w_{3} \\
w_{4}
\end{array}\right)=\left(\begin{array}{c}
M_{1}+\frac{6 E I_{1} v_{2}}{L^{2}}-\frac{4 E I_{1} w_{1}}{L}-\frac{2 E I_{1} w_{2}}{L} \\
V_{2}-\frac{12 E I_{1} v_{2}}{L^{3}}+\frac{6 E I_{1} w_{1}}{L^{2}}+\frac{6 E I_{1} w_{2}}{L^{2}} \\
M_{2}+\frac{6 E I_{1} v_{2}}{L^{2}}-\frac{2 E I_{1} w_{1}}{L}-\frac{4 E I_{1} w_{2}}{L} \\
V_{3} \\
M_{3} \\
M_{4} \\
V_{1}+\frac{12 E I_{1} v_{2}}{L^{3}}-\frac{6 E I_{1} w_{1}}{L^{2}}-\frac{6 E I_{1} w_{2}}{L^{2}} \\
V_{4}
\end{array}\right)=D_{2}
$$


In Eq. (5), it can be seen that the unknowns observed from the previous recursive step are absorbed in both $B_{2}$ and $D_{2}$. The null space, $V_{n 2}$, of the updated matrix $B_{2}$ and the general solution of Eq. (5), $z_{2}$, are given as:

$z_{2}=z_{p 2}+V_{n 2} \cdot \boldsymbol{\rho}_{2}=\left(\begin{array}{c}E I_{2} \\ E I_{2} v_{3} \\ E I_{2} w_{3} \\ v_{3} \\ w_{3} \\ w_{4}\end{array}\right)_{\text {particular }}+\left(\begin{array}{cc}\frac{1}{w_{2}} & 0 \\ \frac{v_{2}+w_{2} L}{w_{2}} & 0 \\ 1 & 0 \\ 0 & -L \\ 0 & 1 \\ 0 & 1\end{array}\right) \cdot\left(\begin{array}{c}\rho_{2,1} \\ \rho_{2,2}\end{array}\right)$

318

319

320

321

322

323

324

325

326

327

328

329

330

331

332

333

334

335

336

337

338

339

340

341

342

343

344

345

346

347

348

349

350

351

352

353

Again, $z_{2}$ can be expressed as the sum of a particular solution, $z_{p 2}$, and any linear combination of the two bases in the null space, $V_{n 2}$, in which the coefficients are given as $\rho_{2,1}$ and $\rho_{2,2}$. In the null space $V_{n 2}$, as given in Eq. (6) no variable is observable since no null row exists. In other words, the recursive steps end as per the criterion of null row in the null space. However, the main reason of the premature end of the recursive steps is that the constraints, i.e. $E I_{2} v_{3}=E I_{2}$. $v_{3}$ and $E I_{2} w_{3}=E I_{2} \cdot w_{3}$, are not incorporated into the process. This is illustrated in a geometric way in Figure 2.a. In this figure, the abscissa in $\rho_{1}$-axis and the ordinate in $\rho_{2}$-axis, indicate the values of the two coefficients $\rho_{2,1}$ and $\rho_{2,2}$ in Eq. (6).

With a given set of $\rho_{2,1}$ and $\rho_{2,2}$, the value of each unknown are specified by Eq. (6) and can be represented by dots in the space in Figure 2.a. To present the value of all unknowns in the same range, these values are normalized by the accurate values obtained from SAP $2000^{[60]}$. The solution of this problem is represented as the point in Figure 2.a, where the values of all normalized unknowns are one. If infinite sets of $\rho_{1}$ and $\rho_{2}$ are provided, all the possible normalized values obtained by these sets will yield six planes. These planes are shown in Figure 2.a. For a given set, the equation $\left\{\rho_{1}=\rho_{2,1}, \rho_{2}=\rho_{2,2}\right\}$ specifies a vertical line where $\mathrm{z}$ can take any value and the intersections of this vertical line with the six planes indicate a specific solution of Eq. (5). For illustration, when $\rho_{2,1}=-22.00, \rho_{2,2}=4.20 \times 10^{-4}$, as indicated by the vertical line in Figure 2.a, it can be seen that the 6 solutions are deviated from the solution to the problem. When the parameters are chosen as $\rho_{2,1}=-22.22, \rho_{2,2}=4.12 \times 10^{-4}$, the six intersections of the vertical line and the six planes will occur at the solution of the problem. To clarify this, the variations of the normalized solution against $\rho_{2.1}$ with fixed $\rho_{2.2}$ of $4.12 \times$ $10^{-4}$ and against $\rho_{2.2}$ with fixed $\rho_{2.1}$ of -22.22 , respectively, are plotted in Figure 2.b and 2.c. Evidently, the solution of the problem comes from a particular solution in the general solution. However, SSI by OM is incapable of detecting this solution in the general solution. The reason is that observability treats the coupled unknowns, $\left\{E I_{2} v_{3}, E I_{2} w_{3}\right\}$, as independent of the corresponding component variables, $\left\{E I_{2}, v_{3}, w_{3}\right\}$ though they should satisfy the constraints of $\left\{E I_{2} v_{3}=E I_{2} \cdot v_{3}, E I_{2} w_{3}=E I_{2} \cdot v_{3}\right\}$. In Figure 2.b and 2.c, these constraints are gradually satisfied by adjusting $\rho_{2,1}$ and $\rho_{2,2}$.

Consider the FEM of a one-story, one-bay frame depicted in Figure 3. In this frame, each column and each beam are divided into two elements. The end nodes of the columns are clamped. The possible measurements within this structure include 5 horizontal deflections, 5 vertical deflections and 5 rotations. For simplicity, it is assumed that the areas of the columns and the beam are known, and the flexural inertias of the columns are identical. Hence, the two unknowns here are the flexural stiffnesses of the columns and the beam. Due to the requirement of essential set, two measurements should be used and sufficient to identify the two unknown flexural stiffnesses. However, it is found that measuring any two displacements out of the 15 
354 possible measurements, which is $C_{15}^{2}=105$ possible combinations, cannot ensure the 355 identifiability of the two parameters. Half of the possible measurement sets can only identify 356 one flexural stiffnesses and the other half cannot identify any parameter. This means that no 357 essential set exists in this structure. A closer inspection of the general solution for this structure 358 shows that the constraints between the variables are also missing.

359 From the simply-supported beam example, an explanation for the observability method being 360 incapable of ensuring identifiability is provided algebraically. The algebraic analysis shows that 361 the nonlinear constraints between the unknowns are neglected in the traditional OM. In the 362 graphical illustration of the general solution of Eq. (6), it is shown that the exact solution is 363 obtained when the constraints are satisfied. Moreover, an example of a frame is given to clarify 364 that essential set may not exist in some structures due to the same reason.

365 In order to get more knowledge of this phenomenon, an exhaustive examination of observability 366 of the structural parameters is carried out in a slightly more complicated structure depicted in 367 Figure 4.a. This is a $15 \mathrm{~m}$ simply supported beam with 5 evenly divided elements. A vertical 368 concentrated force is applied downwards at node 3 with a magnitude of $100 \mathrm{kN}$. The flexural 369 stiffnesses of the five elements are deliberately chosen as distinct values of $1.5 \times 10^{6} \mathrm{kN}$. $370 \mathrm{~m}^{2}, 1.2 \times 10^{6} \mathrm{kN} \cdot \mathrm{m}^{2}, 1.1 \times 10^{6} \mathrm{kN} \cdot \mathrm{m}^{2}, 1.4 \times 10^{6} \mathrm{kN} \cdot \mathrm{m}^{2}$ and $1 \times 10^{6} \mathrm{kN} \cdot \mathrm{m}^{2}$. In this 371 structure, potential measurements include six rotations, $\mathrm{w}_{1} \sim \mathrm{w}_{6}$, and four vertical deflections, $372 v_{2} \sim v_{5}$, which are ten in total. All the exact displacements are calculated in SAP 2000 and used 373 as the input in SSI by OM. In the identification problem, the five flexural stiffnesses, from $E I_{1}$ 374 to $E I_{5}$, are assumed as unknown, i.e. $N_{F}=5$, whereas the axial stiffnesses are disregarded due to 375 the load case, i.e. $N_{A}=0$, as assumed before. Therefore, 5 measurements will suffice the 376 requirement of the essential set on the number of measurements.

377 In this structure, the full observability is defined as the ability to identify the 5 unknown flexural 378 stiffnesses. Prior to further discussion, the peculiarity of SSI by OM is qualitatively illustrated 379 by an observability analysis performed on an empirically chosen essential measurement set. In 380 the authors' experience, one straightforward essential set for this structure is measuring all the 381 vertical deflections plus one rotation. For instance, measuring the four vertical deflections $382 v_{2} \sim v_{5}$ and the rotation $w_{1}$. The observability flow (the identification sequence of the unknowns) 383 for this set is provided in Figure 4.b. In this figure, the measurements are enclosed by solid 384 boxes whereas the estimates are enclosed by dashed boxes. It can be seen that the whole 385 structure is identified through 4 recursive steps. In recursive step 1, the flexural stiffness of element $1, E I_{1}$, and rotation of node $2, w_{2}$ are identified first. Meanwhile, the vertical reactions, $V_{1}, V_{5}$, are also obtained directly since this is a statically determinate structure. Furthermore, the estimation of the remaining rotation of element $1, w_{2}$, is used to update the original measurement set and to initiate the subsequent recursive identification process. In recursive step $2, E I_{2}$ and $w_{3}$ can be obtained by observability with two measurements, $v_{2}$ and $v_{3}$, and the estimated rotation of node $2, w_{2}$. Likewise, $\left\{E I_{3}, w_{4}\right\},\left\{E I_{4}, w_{5}\right\}$ and $\left\{E I_{5}, w_{6}\right\}$ are observed in pairs consecutively in recursive steps 3,4 and 5 . Thus, the 5 inertias are all identified progressively through recursive step 1 to recursive step 5 .

In Figure 4.b, the estimation obtained in each step is given in dashed box. It should be noted that the identification of each flexural stiffness is accompanied by the identification of a displacement. The identification of the flexural stiffness and associated displacement is based on the three DOFs of that element, which might be three measurements like step 1, or two 398 measurements plus one estimation of displacement like steps $2 \sim 5$. That is to say, the 
information of the remaining DOF, which is referred as the estimated displacement here, is somehow contained in the other three DOFs. Thus, redundancies might arise in the measurement set if the information of the four displacements of the same element is provided by real-life measurements and/or those estimated displacements. This will be exemplified and clarified in the following paragraph.

In the essential set proposed in Figure 4.b, the redundancy of the measurements is avoided by empirical (artificial) selection of the measurement set. To check the possibility of detecting essential set with no special attention paid to avoid redundancy, an exhaustive investigation of all potential essential sets, which should have 5 measurements, is carried out by SSI by OM. As mentioned before, potential measurements include the four vertical deflections, $v_{2} \sim v_{5}$, and six rotations, $w_{1} \sim w_{6}$. All the essential sets should be composed of 5 out of these 10 potential measurements, resulting in $C_{10}^{5}=252$ possibilities. As clarified by the first example, the same number of measurements as the number of unknowns does not necessarily mean that the structure is fully observable. Bearing this in mind, it is interesting to know how many sets out of these 252 sets can achieve full observability. The same procedure presented in the first example is carried out on all of these 252 enumerated measurement sets. The number of identified flexural stiffnesses by $\mathrm{OM}, N_{F, O M}$, for each set are collected. As there are five unknown flexural stiffnesses, $N_{F, O M}$ might take the value of $1,2,3,4$ and 5 . The frequency of the occurrence of each case is given as the histogram in Figure 5.

418

419

420

421

422

423

424

425

426

427

428

429

430

It can be seen that the occurrence of full observability, $N_{F, O M}=5$, occupies $25.4 \%$ of the 252 sets whereas the occurrence of partial observability, $N_{F, O M}<=4$, occupies $74.6 \%$ of the 252 sets. To distinguish among the sets achieving different levels of observability, they are classified into different patterns with regard to the physical location of the measurements. This can be carried out by taking the subscript of the measurement, i.e. the node number, which indicates the location of the measurement. For instance, assume three measurement sets: (1) $\left\{w_{1}, v_{2}, w_{2}, v_{3}, w_{3}\right\}$; (2) $\left\{w_{1}, v_{2}, v_{3}, v_{4}, w_{4}\right\}$; (3) $\left\{w_{1}, w_{2}, w_{3}, v_{4}, w_{4}\right\}$. These three sets are, respectively, classified as $\{1,2,2,3,3\},\{1,2,3,4,4\}$ and $\{1,2,3,4,4\}$. From the last 2 sets, it can be seen that no distinction is made between measuring the rotation and measuring the deflection of a node. If the same number shows twice in a pattern, then it is saying that both the deflection and the rotation of the node associated with that number are measured. Since the vertical deflection of node 1 and node 6 are null (boundary conditions), $v_{1}$ and $v_{6}$ will be added to each pattern automatically. To understand the relationship between the location of the measurement and the number of identified parameters, the most representative patterns are listed in Table 1.

All the patterns related with $N_{F, O M}=5$, i.e. full observability, are listed in the first column of Table 1 . Note that the empirically chosen measurement set adopted to illustrate the observability flow belongs to the first pattern, $\{1,1,2,3,4,5,6\}$. As indicated by the indices of these patterns, the measurements yielding full observability are taken at physically dispersed locations. These patterns, or the geometrically distributed placement of sensors, maintain the observability flow and thus the full observability is achieved.

In comparison, if the measurements are taken in an intensive way at a local area, redundancy in the measurement set will emerge. For instance, assume the measurement set of $\left\{v_{3}, w_{3}, v_{4}, w_{4}, w_{6}\right\}$, which is the first pattern in the column of $N_{F, O M}=1$ in Table 1 . This set measures all the displacements of element 3 and thus the identification of $E I_{3}$ is as expected. Nevertheless, due to the improper selection of measurements, the observability flow is 
terminated. In SSI by OM, the recursive process is maintained by continuously providing information of the displacements of the nodes which are adjacent to the previously identified elements. To identify the flexural stiffness of the elements next to the element 3 , e.g. $E I_{2}$, apart from the known $v_{3}$ and $w_{3}$, at least one measurement or the estimation of $v_{2}$ or $w_{2}$ is required. Due to the violation of this requirement, the identification of $E I_{2}$ is not executed. Similarly, the identification of $E I_{4}$ is not executed due to the lack of any information of $v_{5}$ or $w_{5}$. The identification of $E I_{5}$, which should follow the identification of $E I_{4}$ in this set, is not executed either. This renders the available information of $w_{6}$ and $v_{6}$ unutilized and ineffective. By analogy, the information of $v_{1}$ is also ineffective due to the failure in the identification of $E I_{1}$. From the previous analysis, it can be seen that the effectiveness of a measurement is defined in the context of a particular measurement set and might be different for another set. These unutilized measurements are referred as ineffective measurements and all of them are given in bold in Table 1. From this measurement set, it can be deduced that one reason for partial observability is that the number of effective measurements is less than the number of unknowns.

However, in the last 2 patterns in the column of $N_{F, O M}=3$, all the measurements are involved in the identification but they still lead to partial observability. In fact, the defect of these patterns $\left\{v_{1}, w_{1}, v_{2}, v_{3}, v_{5}, v_{6}, w_{6}\right\}$, which corresponds with the 5th pattern of $N_{F, O M}=3$, the identifications of the pairs of $\left\{E I_{1}, w_{2}\right\}$ and $\left\{E I_{5}, w_{5}\right\}$ are, respectively, enabled by the information of $\left\{v_{1}, w_{1}, v_{2}\right\}$ and $\left\{v_{5}, v_{6}, w_{6}\right\}$. Likewise, the identification of $I_{2}$ and $w_{5}$ is enabled by the information of $\left\{v_{2}, v_{3}, w_{2}\right\}$. Hence, the up-to-date information of the measurements is composed of $\left\{v_{1}, w_{1}, v_{2}, w_{2}, v_{3}, w_{3}, v_{5}, w_{5}, v_{6}\right.$ and $\left.w_{6}\right\}$. However, the lack of any information of $v_{4}$ or $w_{4}$ terminates the observability flow, and thereby fails the identification of both $E I_{3}$ and $E I_{4}$, despite of the available information of $v_{3}, w_{3}, v_{5}, w_{5}$. Truthfully, this cannot be resolved without introducing any new information in SSI by OM.

With respect to $N_{F, O M}=1$ and 2, all the patterns yielding partial observability can be categorized as cases of the ineffective measurements or of the premature end of the recursive steps. It should be noted that the case of $N_{F, O M}=4$ does not exist. The reason is that if $N_{F, O M}=4$, the direct measurements and those estimated ones which come together with the identifications of these four inertias will certainly yield the full observability.

473

474

475

476

477

478

479

480

481

482

483

484

485

\section{Section 3: SSI by COM}

From the two examples in section 2, it is found that the nonlinear constraints among the unknowns is lacking, and the two reasons for the partial observability are: (1) the premature end of the recursive steps and (2) the ineffective measurements due to redundancy in the measurement sets. It is expected that the supplement of the nonlinear constraints to SSI by OM might improve the performance of the original method in dealing with partial observability. Therefore, SSI by COM, which incorporates the nonlinear constraints through an optimization routine with SSI by OM, is proposed with the aim of exploiting the information contained in the measurements to the maximum extent. The following points have to be taken into account when implementing the optimization:

\subsection{The condition when the optimization should be applied}

Whenever is possible, appropriate measurement sets have to be chosen in order to avoid optimization. It is less desirable to employ COM than just OM due to the fact that the 
computation cost is normally higher for the first option. When optimization is required, SSI by OM will be carried out first and then the general equation from the last step of SSI by OM will be used for COM.

489 3.2 Decoupling of the unknowns and generating the new unknown

490

491

492

493

494

495

496

497

498

499

500

501

502

503

504

505

506

507

$\epsilon=B^{*} \cdot z^{*}-D$

508

509

510

511

512

513

514

515

516

517

518

519

\subsection{Constraints} given in Eq. (8).

As mentioned before, the unknowns in Eq. (2) can always be of two types: (1) monomials of degree one, or single unknowns, $V_{S}$, and (2) monomials of degree two, or coupled unknowns, $V_{c}$. The single unknowns, $V_{s}$, contains flexural stiffnesses, $E I_{j}$, axial stiffnesses, $E A_{j}$, or the displacements, $u_{i}, v_{i}$ or $w_{i}$. The unknowns $V_{c}$ are coupled with some of $V_{s}$ and have to be uncoupled in two steps. In first step, the existent single variables, $V_{S 1}$, in $z$ are picked out first. Subsequently, $V_{c}$ are decomposed into two single unknowns, and then a comparison between these single unknowns and the existing $V_{s 1}$ is carried out. If there exists any new single unknown, which is always the case, these new variables will be designated as $V_{s 2}$. These new single unknowns are added to the unknowns $z$ to form the new unknowns $z^{*}$, i.e. $z^{*}=z \cup V_{s 2}$. For instance, in the case of $z=\left\{E I_{2} w_{2}, E I_{2} w_{3}, E I_{2} v_{3}, E I_{2}\right\}$, coupled unknowns $V_{c}$ include $E I_{2} w_{2}, E I_{2} w_{3}$ and $E I_{2} v_{3}$. The components of $E I_{2} w_{2}, E I_{2} w_{3}$ and $E I_{2} v_{3}$ are $E I_{2}, w_{2}, w_{3}$ and $v_{3}$. Since $E I_{2}$ exists in z, it is categorized as $V_{s 1}$ whereas $w_{2}, w_{3}$ and $v_{3}$ are categorized as $V_{s 2}$. The new unknown $z^{*}$ is $\left\{E I_{2} w_{2}, E I_{2} w_{3}, E I_{2} v_{3}, E I_{2}, w_{2}, w_{3}\right.$ and $\left.v_{3}\right\}$.

\subsection{Rearrangement of the equation $B \cdot z=D$}

To consider the additional unknowns $V_{s 2}$ in $z^{*}$, an adaption on the coefficient matrix B of Eq. (2) is made. A null matrix, $\Omega$, is added to the matrix $\mathrm{B}$ and thereby $V_{s 2}$ is included in $z^{*}$ without violating the equation. In addition, during the optimization, it is inevitable to have residuals in these equations. Thus, a more realistic form of the system of equations is given as:

where $B^{*}=\left[B^{N_{e q} \times N_{z}} \mid \Omega^{N_{e q} \times N_{s 2}}\right], z^{*}=\left\{\begin{array}{c}z^{N_{z} \times 1} \\ V_{s 2}^{N_{s 2} \times 1}\end{array}\right\}, \epsilon$ is a residual vector with a dimension of $N_{e q}$ times $1 . \Omega$ is a null matrix with the dimension of $N_{e q}$ times $N_{s 2}$. The matrix B, and vectors z and D are directly obtained from the system of equations in the last step of SSI by OM. Here, $N_{e q}$ and $N_{z}$, respectively, denotes the number of equations and the number of unknowns in this system. $N_{s 2}$ denotes the number of new single unknowns in $V_{s 2}$.

When the decoupling of $V_{c}$ is accomplished, the constraints between $V_{c}$ and relevant $V_{s}$ are applied by imposing the equality between the value of $V_{c}$ and the product of the values of corresponding $V_{S}$ in the optimization. For the same example presented in 3.2, the matrix form of the corresponding constraints, i.e. $E I_{2} w_{2}=E I_{2} \cdot w_{2}, E I_{2} w_{3}=E I_{2} \cdot w_{3}$ and $E I_{2} v_{3}=E I_{2} \cdot v_{3}$, is

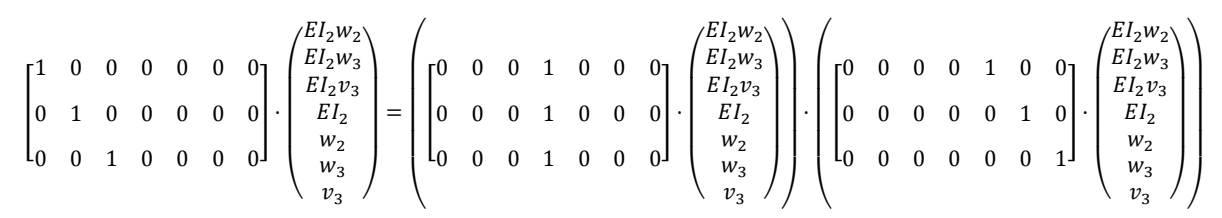


520 The objective function is defined as minimizing the square sum of the residuals in Eq. (7), 521 which is expressed as:

$\min \left(\sum_{k=1}^{N_{e q}} \epsilon_{k}^{2}\right)$

$522 \epsilon_{k}$ is the $k^{\text {th }}$ component of the residual vector $\epsilon$ in Eq. (7).

$523 \quad 3.6$ Other remarks

524 In the optimization, the active-set algorithm ${ }^{[61]}$ is adopted to find the optimal solution to satisfy 525 Eq. (9) and the optimization toolbox of the commercial package Matlab ${ }^{[58]}$ is used to implement 526 this program. For the sake of better convergence and computational efficiency, as indicated by 527 Eq. (10), the element in row $i$ and column $k$ of the coefficient matrix $B^{*}$ in Eq. (7), $B_{i, k}^{*}$, will be 528 multiplied by the numerical value of the $k^{\text {th }}$ unknown obtained from direct stiffness method, $\overline{z_{k}^{*}}$,

$\overline{\overline{B_{l, k}^{*}}}=B_{i, k}^{*} \cdot \overline{z_{k}^{*}}, \quad \forall i, k=1,2, \cdots,\left(N_{z}+N_{s 2}\right)$

529 The vector $D$ in Eq. (7) will remain the same. Consequently, in Eq. (11), the corresponding 530 value of the solution minimizing the objective function, will be normalized by $\overline{z_{k}^{*}}$, and thus the 531 numerical value of the normalized unknowns, $\overline{\overline{z_{k}^{*}}}$, are supposed to be around 1. However, in case 532 of some very small or zero values of $\overline{\overline{z_{k}^{*}}}$, which might lead to instability of the solution, a 533 threshold $z_{t h}$ of $10^{-6}$ is chosen for $\overline{\overline{z_{k}^{*}}}$. If the value of $\overline{\overline{z_{k}^{*}}}$ is larger than the selected threshold, 534 the original value will be used directly in Eq. (10); otherwise, the threshold will be used in Eq. 535 (10).

$\overline{\overline{z_{k}^{*}}}=z_{k}^{*} / \overline{z_{k}^{*}}, \quad k=1,2, \cdots,\left(N_{z}+N_{s 2}\right)$

$\overline{z_{k}^{*}}= \begin{cases}z_{k}^{*} & \text { if } \overline{z_{k}^{*}} \geq z_{t h} \\ z_{t h} & \text { if } \overline{z_{k}^{*}}<z_{t h}\end{cases}$

536 For this reason, an intuitive guess for $\overline{\overline{z_{k}^{*}}}$ of all ones will be used to initialize the optimization. A 537 concurrent advantage of this guess is that the nonlinear constraints are satisfied automatically 538 since the product of ones is always one. It is worth well doing so due to the fact that initial 539 values satisfying or closely satisfying many of the constraints reduce the work involved in 540 finding a first feasible solution. Nevertheless, regardless of the initial values recommended here, 541 the same solution might be attained at a higher cost of time and computation capacity if this 542 normalization is not carried out, which is verified later. In addition, the lower bound and the 543 upper bound for each element in $\overline{\overline{z_{k}^{*}}}$, respectively, are set as 0.1 and 10.

$544 \quad 3.5$ Proposed algorithm

545 This proposed method combines SSI by OM with optimization and thereby includes the 546 nonlinear constraints in the identification process. The algorithm for SSI by COM is 547 summarized as follows:

548 Step1: Apply SSI by OM and check whether the full observability is achieved or not. 549 Initiate SSI by OM with the given measurement set. Form the general equation (Eq. (2)), and 
check the null space of coefficient matrix B to see if any parameters are observed. If so, update the input by incorporating observed variables and reinitiate the previous procedure until the end of the recursive process, i.e. no new variable is observable. If not, the $\mathrm{OM}$ is ended without estimating any unknown. If full observability is achieved at the end of the recursive process, i.e. the structure is fully observable, it is not necessary to perform optimization; otherwise, go to step 2. It is highlighted that several recursive steps can be done in step 1 until no further unknowns are observable by $\mathrm{OM}$;

Step2: Obtain the equation $\boldsymbol{B} \cdot \boldsymbol{z}=\boldsymbol{D}^{[33,57]}$. Extract the updated general equation $B \cdot z=D$ from the last step of SSI by OM.

Step3: Analyze the unknowns $z$ and generate the new unknowns $z^{*}$. Divide $z$ into $V_{c}$ and $V_{s 1}$. Then compare every component of the coupled unknowns $V_{c}$ with the existent single unknowns $V_{s 1}$ and collect the single unknowns $V_{s 2}$ which were not present in $V_{s 1}$. Generate the new unknowns $z^{*}$ by adding the former unknowns $z$ and the additional single unknowns $V_{s 2}$.

Step4: Form the new matrix $B^{*}$. Analyze the dimension of matrix B and append the null matrix $\Omega$ to contain $V_{S 2}$ in the $z^{*}$ without violating the equations.

Step5: Obtain the normalized unknown $\overline{\overline{z^{*}}}$. Multiply the column of the matrix $B^{*}$ with the expected value $\overline{z^{*}}$ determined by Eq. (12) so as to obtain the $\overline{\overline{B^{*}}}$ and the normalized unknown $\overline{z^{*}}$.

Step6: Store the constraint information. Check every element in $\overline{\overline{z^{*}}}$ and build the matrix that relates the nonlinear relationship between $V_{c}$ and $V_{S}$.

Step7: Optimization. Choose the initial values of $\overline{\overline{z^{*}}}$ and set the bounds for the solution. The objective is to minimize the square sum of the residual vector, $\epsilon$. In the optimization process, the nonlinear constraints are imposed by ensuring the equality between the coupled unknowns $V_{c}$ and the product of corresponding single unknowns $V_{s}$.

573 A summary of the procedure is shown in the flow chart in Figure 6.

\section{3.6. Application of the algorithm}

First, both structure depicted in Figure 1 and Figure 3 are re-analyzed by COM. As expected, 576 after the application of COM, the first example becomes identifiable with the given 577 measurement set. Also, 45 out of the 105 measurement sets becomes capable of identifying all parameters for the frame. However, these structures are far from proving the strength and robustness of COM. In order to do this, the observability of the structure depicted in Figure 4.a is re-analyzed by the constrained observability method.

When COM is applied in this structure, the influence of the normalization and the choice of initial values on the accuracy of the result of the optimization are checked here. Note that the flexural stiffnesses of the five elements are deliberately chosen as distinct values of $\{1.5 \times$ $10^{6} \mathrm{kN} \cdot \mathrm{m}^{2}, 1.2 \times 10^{6} \mathrm{kN} \cdot \mathrm{m}^{2}, 1.1 \times 10^{6} \mathrm{kN} \cdot \mathrm{m}^{2}, 1.4 \times 10^{6} \mathrm{kN} \cdot \mathrm{m}^{2}$ and $\left.1 \times 10^{6} \mathrm{kN} \cdot \mathrm{m}^{2}\right\}$. Instead of normalizing the columns of the matrix B with the real inertias, these columns are normalized by $1 \times 10^{6} \mathrm{KN} \cdot \mathrm{m}^{2}$. Therefore, the normalized estimate of these inertias should be $[1.5,1.2,1.1,1.4,1]$. Meanwhile, instead of using all ones as the initial values, random numbers generated by uniform distribution on [0.8,1.2] are used. It should be noted that some of the normalized estimates do not lie in the sampling interval of this distribution. According to the 
result, the optimization still converges to the same solution as that obtained by using the recommended normalization and initial values but with more iterations in the computation. In the following comparison, the normalization factor and the initial values are taken as recommended. The frequency of occurrence of the number of observed flexural stiffnesses by COM, $N_{F, C O M}$, equal to 1 to 5 is presented in Figure 7 .a.

Comparing the results presented in Figure 5 and Figure 7.a, a drastic increase, in the number of the measurement sets yielding full observability, from 64 for SSI by OM to 162 for SSI by COM is seen. In fact, all of the previous 64 sets by OM are contained in the new 162 sets by 598 COM due to the equivalence of the step 1 (recursive process) in SSI by COM and SSI by OM. 599 In other words, the improvement of the observability level occurs in the remaining 188 (=252plotted in Figure 7.b. For better visualization, the measurement sets are sorted so as to cluster those sets with the same value of $\mathrm{N}_{\mathrm{F}, \mathrm{OM}}$ in an ascending order. In this figure, the number of identified flexural stiffnesses, $\mathrm{N}_{\mathrm{F}}$, for different measurement sets by $\mathrm{COM}$ and $\mathrm{OM}$ are, respectively, represented by circles and points. The abscissa of the markers is the numbering of the sets whereas the ordinate is the number of identified flexural stiffnesses. If an increase in the number of identified parameters is attained by COM, then a position of the circle higher than the one of the dot for that measurement set should be expected. It can be seen that the majority of the sets yielding $N_{F, O M}=3$ by OM, alter to yield $N_{F, C O M}=5$ by COM. With regard to these measurement sets, it is found that the discontinuity of the observability flow can be overcome by applying the nonlinear constraint. For instance, in the final step of the observability analysis on the measurement set $\left\{v_{1}, w_{1}, v_{2}, v_{3}, v_{5}, v_{6}, w_{6}\right\}$, which was used previously, the up-to-date information, apart from the original measurements, contains the estimated displacements $\left\{w_{2}, w_{3}, w_{5}\right\}$ and flexural stiffnesses $\left\{E I_{1}, E I_{2}, E I_{5}\right\}$. Due to the lack of the information of $w_{4}$ or $v_{4}, E I_{3}$ and $E I_{4}$ could not be identified despite the information of $\left\{v_{3}, w_{3}, v_{5}, w_{5}\right\}$. Nevertheless, with the help of the nonlinear constraint, the structure can always be solved in this case. An explanation for this is that the compatibility requirement of the displacements at the mutual node of these two adjacent elements, i.e. the displacements of the node 4 , and the imposition of the nonlinear constraints force the solution. This deduction can be justified by another case. From the result of the set $\left\{v_{1}, w_{1}, v_{3}, w_{3}, v_{5}, w_{6}, v_{6}\right\}$ by OM, a poor performance, $N_{F, O M}=1$, of this set occurs since merely the identification of $\left\{w_{5}, E I_{5}\right\}$ is attainable. It should be noticed that the condition of the previous deduction is satisfied here for both pairs of elements 1,2 and elements 3,4 since all the displacements of nodes 1,3,5 are known. As expected, this set yields the full observability by COM, which further verifies the deduction. In effect, each measurement set shifting from partial observability towards full observability, due to the adoption of COM, has been checked in an exhaustive way. It is found that the observability flow of each of these sets are maintained by the same mechanism. Moreover, for the same reason, three measurement sets, though not yielding the full observability, identify more inertias, switching from the case of $N_{F, O M}=1$ to the case of $N_{F, C O M}=3$.

Generally, measurements can be regarded as an approach to get information of the static response of the structure, i.e. displacements. In the context of essential measurements, if some of the sensors are placed too intensively at a local area, then the other sensors will be placed sparsely in the remaining areas. This leads to the loss of information of those areas, or leads to the partial observability. Bearing this in mind, a reexamination has been performed on those patterns where no improvement is obtained with COM. For instance, if the information obtained by measurement is considered as a geometric constraint on the deflection shape of the structure, 
636 the estimation of the parameters $E I_{1}, E I_{2}$ and $E I_{3}$ is not fixed in the pattern $\{1,1,4,4,5,6,6\}$. That 637 is, a relation can be established among them but it is impossible to get a unique estimate of these 638 parameters.

639 From the comparison of the results of observability analyses obtained by OM and by COM, it 640 can be seen that COM, as an extended version of OM, enhances the performance of the original 641 method. The number of essential sets soars from 64 to 162 . Nevertheless, if the sensors are 642 placed too intensively, there still exists possibility of partial observability. Hence, distributed 643 placement of sensors is strongly recommended.

644 In the preliminary stage of condition assessment of any structure, the location of the possible 645 measurements can be determined according to the accessibility of the location. The final 646 location of the measurements will be a specific combination of some possible measurements. 647 Prior to instrumentation, the capability of a given measurement set to identify all parameters in 648 the structural system will be examined using the COM. Among all possible measurements sets, 649 the ones more likely to produce full observability are the ones that include at least one 650 measurement for every mechanical property at the location of the element with the unknown 651 parameter to be identified.

\section{Section 4: Application in a building structure}

653 To test the performance of the proposed method, a large structure ${ }^{[33]}$ previously analyzed by OM 654 is re-analyzed by COM. This structure is a 13-storey frame with a height of $39 \mathrm{~m}$ and a width of $65532 \mathrm{~m}$. This structure is modelled by beam elements with three DOFs per node in SAP2000. 656 Eight different sections (from I to VIII) of different inertias and different areas (see Table 2) are 657 used to model the structure and Young's modulus is assumed as $35000 \mathrm{MPa}$. The geometry of 658 the structure and the load case is described in Figure 8. Also, element properties are indicated by 659 Roman numerals in this figure. However, in the following analysis, each of these 16 mechanical 660 parameters is perturbed by random numbers. Apart from the choice of the values of the 661 parameters, another major difference between the previous analysis and the current one lies in 662 the number of measurements. At that time, 156 vertical deflections were measured. In the 663 current analysis, the number of measurements are chosen the same as the number of unknowns. 664 Also, the suggestion of distributed placement of sensors is followed here and the locations of 665 these sensors are indicated by crosses in Figure 8. To illustrate the robustness of COM, 4 sets of 666 the 16 mechanical parameters are synthesized by the product of the intact values and random 667 numbers evenly distributed on $[0.8,1.2]$, referred as perturbation factors later. The nodal 668 displacements calculated by SAP2000 using these 4 generated parameter sets are used as the 669 input of SSI by COM. Initial values of all ones are used here. Regarding the results from COM, 670 the estimates of axial stiffnesses (flexural stiffnesses) are normalized by the product of Young's 671 modulus and the intact areas (inertias). In other words, an accurate estimation is characterized 672 by the closeness between these normalized estimates and the perturbation factors. The 673 normalized estimates and the perturbation factors for the axial stiffnesses and flexural 674 stiffnesses are given in Figure 9.a and 9.b. In these two figures, it can be seen that the estimates 675 are close to the perturbation factors. Their ratios are also provided in Figure 9.c. In this figure, 676 for each of these parameters, the deviation of the ratio between the estimate and the true value is 677 within 2 percent, which is acceptable. It should be also noted that by OM, no inertia or area can 678 be identified whereas all these parameters are obtainable by COM. 
679 This example shows the applicability of SSI by COM presented in this paper and its ability to 680 identify the mechanical parameters of a large structure using essential sets.

681 In this example, the structural system is defined by 16 structural parameters. One concern is that 682 this assumption will lead to an average estimate of the structural parameters, which might not 683 identify some localized damage appropriately. However, the modelling assumption that the 684 flexural stiffness and the axial stiffness of each element being different in such a structure could 685 be a formidable task for the proposed method to solve. In order to deal with the trade-off 686 between the issue of the number of parameters and the computation cost, it is advisable to use 687 some engineering techniques, e.g. visual inspections, acoustic emission or impact method 688 (hammering), before making modelling assumption with the aim of reducing the unknowns in 689 the model.

690 Conclusion:

691 One crucial step in SSI is using the adequate number of measurements to achieve full 692 observability of the whole structure. From the formulation of the identification problem by 693 observability, the number of the measurements should be equal to the number of unknowns in 694 the structure. However, measurement sets with this number of measurements do not inevitably 695 lead to the successful identification of the whole structure. This is verified by a simply 696 supported beam with only one unknown. By the analysis of this structure, the lack of nonlinear 697 constraints, which is essentially induced by the linearity of the standard OM, is presented for the 698 first time.

699 To further understand the mechanism, a thorough examination of the observability of structural 700 parameters in a simply supported beam with more unknowns is carried out with respect to 252 701 numerated measurement sets. Before this exhaustive check, SSI by OM is applied on the same structure with an empirically chosen essential set so as to manifest the peculiarity of the method and conceptualize the consecutive observability flow. Subsequently, all the 252 sets are categorized into 70 patterns by the physical location of the measurements and corresponding identification results are discussed with respect to these patterns. It is found that the reasons of partial observability are: (1) the premature end of the recursive steps and (2) the redundant information due to intensive placement of sensors.

To address the issue of partial observability, SSI by COM, where the lacking nonlinear constraints are appended to the original SSI by OM through an optimization routine, is proposed with the aim of fully exploiting the information in measurements. In the first step of the proposed method, SSI by OM is performed until no more parameters are observed. If full observability is achieved, then the algorithm returns. Otherwise, the general equation (Eq. (2)) from the last recursive step will be extracted. The structure of the unknowns in this equation is analyzed first and the relation between variables is identified as the nonlinear constraints used next in the optimization. In the last step of the SSI by COM, an optimization is performed on the observability equation with the acquired nonlinear constraints and all the parameters observed in the first step. The efficacy of the proposed method to improve identifiability is justified by reanalyzing the same structure with the 252 enumerated sets. The number of sets achieving full observability by COM roughly doubles that number by OM. Nevertheless, it is strongly recommended to place the sensor in a dispersed way since the structure is still not fully observable in case of extremely intensive placement of sensors. The strength and robustness of 
perturbed by random numbers. It is seen that the axial stiffnesses and the flexural stiffnesses of all structural members can be estimated accurately.

\section{Acknowledgement}

This method is able to identify mechanical properties for linear systems. For non-linear systems, the load test will induce a deformation increment that will provide the information of secant mechanical properties of the different elements. It is highlighted that COM is not suitable to identify structures with geometrical non-linear behaviour. As the use of an essential set provides the only possible solution that satisfies the equations, measurement or modelling errors will affect the estimates. These effects are not studied in this paper but it will be addressed in the near future. The importance of this research is that the supplement of the nonlinear constraints to the OM fully exploits the information provided by measurements. In contrast with SSI by $\mathrm{OM}$, the range of the measurement sets qualified to be essential sets are greatly enlarged by applying the supplementary constraints via optimization. Besides, in some other sets, even though full observability is not achieved, number of identified parameters are also increased. Furthermore, the sharp decrease in the number of measurements used in the identification of the mechanical parameters in a 13-storey building also justifies the efficacy of the COM.

This work was partially funded by the Spanish Ministry of Economy and Competitiveness and the FEDER fund through the grant project (BIA2013-47290-R) directed by Jose Turmo. It is also to be noted that part of this work was done through a collaborative agreement between Tongji University (China) and Technical University of Catalonia, UPC, BarcelonaTech. This included an exchange of faculty financed by the Chinese government. The financial support from the High End Foreign Experts program (GDW20143100115) from the Chinese government is greatly appreciated. Funding for this research has been provided to Mr. LEI Jun by the Chinese Scholarship Council through its program (No.201506260116) and by the Ministerio de Economia y Competitividad through its program (BES-2014-07022).

\section{References:}

[1] Chisari C, Bedon C, Amadio C. Eng Struct 2015; 102: 80-92.

[2] Lozano-Galant JA, Nogal M, Turmo J, Castillo E. Comput Concr 2015; 15(5): 771-794.

[3] Kao CY, Loh CH. Struct Control Heal Monit 2013; 20(3): 282-303.

[4] Sanayei M, Scampoli SF. J Eng Mech 1991; 117(5): 1021-1036.

[5] Hjelmstad KD, Shin S. J Eng Mech 1997; 123(6): 568-576.

[6] Terlaje A, Truman K. Adv Struct Eng 2007; 10(6): 607-621.

[7] Caddemi S, Morassi A. Int J Solids Struct 2007; 44(16): 5301-5315.

[8] Walsh BJ, González A. Key Eng Mater 2009; 413-414: 269-276.

[9] Pioldi F, Rizzi E. Comput Mech 2016; 58(2): 307-327.

[10] Wang X, Zheng GT. Mech Syst Signal Process 2016; 68-69: 394-415.

[11] Breuer P, Chmielewski T, Górski P, Konopka E, Tarczyński L. Struct Control Heal Monit 2015; 22(7): 1002-1023.

[12] Dowling J, OBrien EJ, González A. Eng Struct 2012; 44: 13-22.

[13] Bakhtiari-Nejad F, Rahai A, Esfandiari A. Eng Struct 2005; 27(12 SPEC. ISS.): 1784-1793.

[14] Sanayei M, Imbaro G, McClain J, Brown L. J Struct Eng 1997; 123(6): 792-798.

[15] Wang C, Huang C, Chen C. Adv Adapt Data Anal 2011; 3(4): 417-445.

[16] Boumechra N. Struct Control Heal Monit 2016: DOI: 10.1002/stc.1972.

[17] Lozano Galant JA. Construction control of cable-stayed bridges. Universitat Politècnica 
de Catalunya, BarcelonaTech, 2013.

[18] Sun Z, Nagayama T, Fujino Y. J Civ Struct Heal Monit 2016; 6(2): 255-264.

[19] Choi IY, Lee JS, Choi E, Cho HN. Comput Struct 2004; 82(29): 2483-2492.

[20] Santos JP, Crémona C, Calado L, Silveira P, Orcesi AD. Struct Control Heal Monit 2015; 23(7): 1047-1069.

[21] Bandara RP, Chan THT, Thambiratnam DP. Eng Struct 2014; 66: 116-128.

[22] Alavi AH, Hasni H, Lajnef N, Chatti K, Faridazar F. Autom Constr 2016; 62: 24-44.

[23] de Lautour OR, Omenzetter P. Struct Control Heal Monit 2010; 17(6): 614-631.

[24] Bao C, Hao H, Li Z. Struct Control Heal Monit 2013; 20(5): 769-788.

[25] Mei L, Mita A, Zhou J. Struct Control Heal Monit 2016; 23(2): 218-236.

[26] Okada R, Nakata N, SPENCER Jr BF, Kasai K, Kim SB. J Earthq Eng 2006; 10(1): 97-125.

[27] Ku CJ, Tamura Y, Yoshida A, Miyake K, Chou LS. Wind Struct An Int J 2013; 16(2): 157178.

[28] Liu W, Gao W cheng, Sun Y. J Vib Acoust 2009; 131(3): 34503.

[29] González A, Covián E, Casero M, Cooper J. Key Eng Mater 2013; 569-570: 1170-1177.

[30] Walsh BJ, González A, Cantero D. The Tenth International Conference on Computational Structures Technology, CST2010, Valencia, Spain, 14-17 September, 2010, Paper 51.

[31] Lam HF, Yang J, Au SK. Eng Struct 2015; 102: 144-155.

[32] Zhang FL, Xiong HB, Shi WX, Ou X. Struct Control Heal Monit 2016; 23(11): 1366-1384.

[33] Lozano-Galant JA, Nogal M, Castillo E, Turmo J. Comput Civ Infrastruct Eng 2013; 28(6): 434-450.

[34] Lei J, Lozano-Galant JA, Nogal M, Xu D, Turmo J. Struct Control Heal Monit 2016. DOI: 10.1002/stc.1923

[35] Castillo E, Conejo AJ, Eva Pruneda R, Solares C. Comput Oper Res 2007; 34(6 SPEC. ISS.): 1708-1720.

[36] Castillo E, Nogal M, Lozano-Galant JG, Turmo J. Math Probl Eng 2016; 2016: 25.

[37] Díaz S, González J, Mínguez R. J Water Resour Plan Manag 2016; 142(4): 4015071.

[38] Nagar AK, Powell RS. Building Partnerships, American Society of Civil Engineers; Reston, VA: 2000, 1-10

[39] Bargiela A. IEE Proc D Control Theory App/ 1985; 132(6): 245.

[40] Castillo E, Conejo AJ, Menéndez JM, Jiménez P. Comput Civ Infrastruct Eng 2008; 23(3): 208-222.

[41] Castillo E, Gallego I, Sanchez-Cambronero S, Rivas A. IEEE Trans Intell Transp Syst 2010; 11(4): 799-813.

[42] Castillo E, Nogal M, Rivas A, Sánchez-Cambronero S. Transp B Transp Dyn 2013; 1(1): 68-102.

[43] Castillo E, Jimenez P, Menendez JM, Conejo AJ. IEEE Trans Intell Transp Syst 2008; 9(2): 275-287.

[44] Caro E, Arévalo I, García-Martos C, Conejo AJ. Electr Power Syst Res 2013; 104: 207-215.

[45] Mahari A, Seyedi H. Electr Power Syst Res 2013; 103: 78-85.

[46] Abur A, Exposito AG. Power system state estimation: theory and implementation. CRC press; Boca Raton, Florida: 2004.

[47] Lozano-Galant JA, Nogal M, Paya-Zaforteza I, Turmo J. Struct Infrastruct Eng 2014; 10(11): 1331-1344.

[48] Lozano-Galant JA, Dong X, Payá-Zaforteza I, Turmo J. Comput Struct 2013; 121: 64-75.

[49] Lozano-Galant JA, Turmo J. Eng Struct 2014; 79: 341-353.

[50] Castillo E, Lozano-Galant JA, Nogal M, Turmo J. J Civ Eng Manag 2015; 21(6): 689-697.

[51] Sanayei M, Saletnik MJ. J Struct Eng 1996; 122(5): 563-572.

[52] Sanayei M, Onipede O, Babu SR. AIAA J 1992; 30(9): 2299-2309.

[53] Cha YJ, Agrawal AK, Kim Y, Raich AM. Expert Syst App/ 2012; 39(9): 7822-7833.

[54] Chang M, Pakzad SN. J Bridg Eng 2014; 19(6).

[55] Jin H, Xia J, Wang Y qiong. J Zhejiang Univ Sci A 2015; 16(6): 464-477. 
1

2

3

4

5

6

7

8

9

10

11

12

13

14

15

16

17

18

19

20

21

22

23

24

25

26

27

28

29

30

31

32

33

34

35

36

37

38

39

40

41

42

43

44

45

46

47

48

49

50

51

52

53

54

55

56

57

58

59

60

821 [56] Malings C, Pozzi M. Struct Saf 2016; 60: 77-90.

822 [57] Nogal M, Lozano-Galant JA, Turmo J, Castillo E. Struct Infrastruct Eng 2015; 12(9): 12168231227.

824 [58] The MathWorks I. Matlab Primer. 2015b ed, https://www.mathworks.com/help/ pdf_doc/matlab/getstart.pdf; 2015.

[59] Incorporated WM. Maple V: learning guide. 3rd ed. Springer-Verlag New York; 1998.

[60] CSI. https://www.csiamerica.com/products/sap2000, 2012.

[61] Nocedal J, Wright SJ. Numerical optimization. Springer Science \& Business Media; 2006.

830

831

832 
833 Table 1. Examples of patterns identifying different number of parameters by OM, and 834 corresponding identified flexural stiffnesses.

\begin{tabular}{|c|c|c|c|c|c|c|c|}
\hline \multirow{2}{*}{\multicolumn{2}{|c|}{$\begin{array}{c}\text { Full observability } \\
\qquad N_{F, O M}=5\end{array}$}} & \multicolumn{6}{|c|}{ Partial Observability } \\
\hline & & \multicolumn{2}{|c|}{$N_{F, O M}=3$} & \multicolumn{2}{|c|}{$N_{F, O M}=2$} & \multicolumn{2}{|c|}{$N_{F, O M}=1$} \\
\hline Pattern & Identified & Pattern & Identified & Pattern & Identified & Pattern & Identified \\
\hline $1,1,2,3,4,5,6$ & $E I_{1} \sim E I_{5}$ & $1,1,2,3,4, \mathbf{6 , 6}$ & $E I_{1} \sim E I_{3}$ & $1,1,2,2,3,3,6$ & $E I_{1}, E I_{2}$ & $1,3,3,4,4,6,6$ & $E I_{3}$ \\
\hline $1,2,2,3,4,5,6$ & $E I_{1} \sim E I_{5}$ & $\mathbf{1 , 1 , 3 , 4 , 5 , 6 , 6}$ & $E I_{3} \sim E I_{5}$ & $1,1,2,2,3, \mathbf{5 , 6}$ & $E I_{1}, E I_{2}$ & $1,1,2, \mathbf{4 , 4}, \mathbf{6}, \mathbf{6}$ & $E I_{1}$ \\
\hline $1,2,3,3,4,5,6$ & $E I_{1} \sim E I_{5}$ & $1,1,2,2,3,4,6$ & $E I_{1} \sim E I_{3}$ & $1,1,2,3,3, \mathbf{5 , 6}$ & $E I_{1}, E I_{2}$ & $1,1,3,3,4,4,6$ & $\mathrm{EI}_{3}$ \\
\hline $1,2,3,4,4,5,6$ & $E I_{1} \sim E I_{5}$ & $1,1,2,3,3,4,6$ & $E I_{1} \sim E I_{3}$ & $1,1,4,5,5,6,6$ & $E I_{4}, E I_{5}$ & $\mathbf{1}, \mathbf{1}, \mathbf{3}, 5,5,6,6$ & $E I_{5}$ \\
\hline $1,2,3,4,5,5,6$ & $E I_{1} \sim E I_{5}$ & $1,1,2,3,5,6,6$ & $E I_{1}, E I_{2}, E I_{5}$ & $\mathbf{1 , 2 , 4 , 4 , 5 , 5 , 6}$ & $E I_{4}, E I_{5}$ & $1,1,2,2, \mathbf{4 , 6 , 6}$ & $E I_{1}$ \\
\hline $1,2,3,4,5,6,6$ & $E I_{1} \sim E I_{5}$ & $1,1,2,4,5,6,6$ & $E I_{1}, E I_{4}, E I_{5}$ & $1,1,2,2,5,6,6$ & $E I_{1}, E I_{5}$ & $\mathbf{1 , 3 , 3 , 5 , 5 , 6 , 6}$ & $E I_{5}$ \\
\hline
\end{tabular}


1

2

3

4

5

6

7

8

9

10

11

12

13

14

15

16

17

18

19

20

21

22

23

24

25

26

27

28

29

30

31

32

33

34

35

36

37

38

39

40

41

42

43

44

45

46

47

48

49

50

51

52

53

54

55

56

57

58

59

60

836 Table 2. Element types of different structural members and associated geometric property

\begin{tabular}{lccccc}
\hline \multicolumn{1}{c}{ Structural members } & Element Type & Areas $\left(\mathrm{m}^{2}\right)$ & \multicolumn{2}{c}{ Inertia $\left(\mathrm{m}^{4}\right)$} \\
\hline outer bottom column & $\mathrm{I}$ & $\mathrm{A}_{1}$ & 0.563 & $\mathrm{I}_{1}$ & 0.026 \\
outer intermediate column & $\mathrm{II}$ & $\mathrm{A}_{2}$ & 0.360 & $\mathrm{I}_{2}$ & 0.011 \\
outer top column & $\mathrm{III}$ & $\mathrm{A}_{3}$ & 0.250 & $\mathrm{I}_{3}$ & 0.005 \\
Interior bottom column & $\mathrm{IV}$ & $\mathrm{A}_{4}$ & 0.360 & $\mathrm{I}_{4}$ & 0.011 \\
Interior intermediate column & $\mathrm{V}$ & $\mathrm{A}_{5}$ & 0.250 & $\mathrm{I}_{5}$ & 0.011 \\
Interior top column & $\mathrm{VI}$ & $\mathrm{A}_{6}$ & 0.160 & $\mathrm{I}_{6}$ & 0.002 \\
Core & $\mathrm{VII}$ & $\mathrm{A}_{7}$ & 1.800 & $\mathrm{I}_{7}$ & 5.400 \\
beam & $\mathrm{VIII}$ & $\mathrm{A}_{8}$ & 0.180 & $\mathrm{I}_{8}$ & 0.005 \\
\hline
\end{tabular}

837 


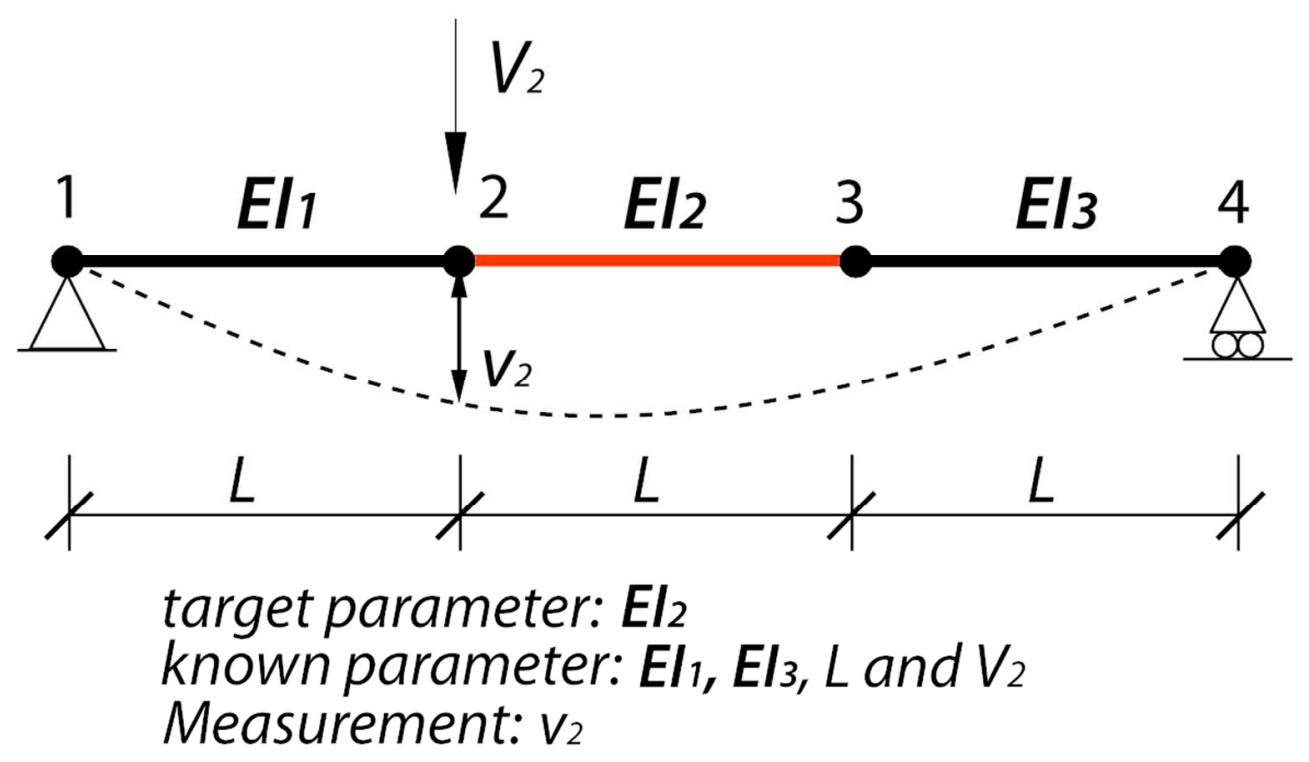

Figure 1. 4-node simply supported beam $100 \times 67 \mathrm{~mm}(300 \times 300 \mathrm{DPI})$ 
(a) 1.06

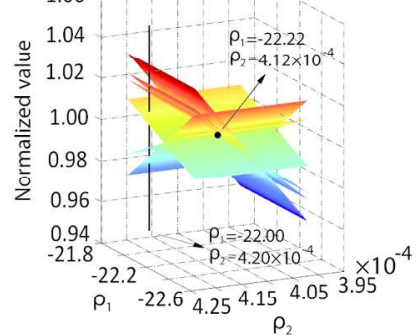

(b)

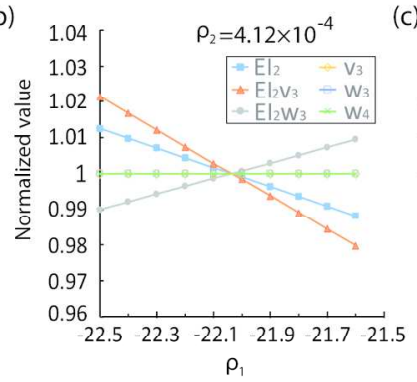

(c)

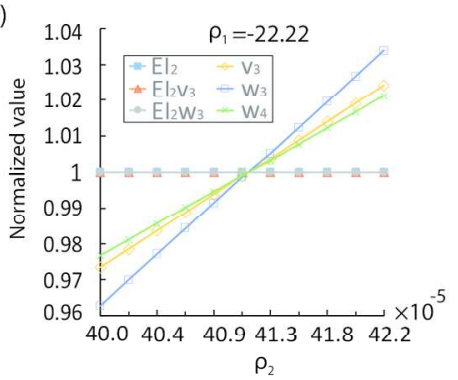

Figure 2. (a) geometric explanation of the null space, (b) variation of the normalized estimates with the variation of $\rho_{1}$ when $\rho_{2}=4.12 \times 10^{-5}$, (c) variation of the normalized estimates with the variation of $\rho_{2}$ when $\rho_{1}=-22.22$

$169 \times 62 \mathrm{~mm}(300 \times 300 \mathrm{DPI})$ 
(a)

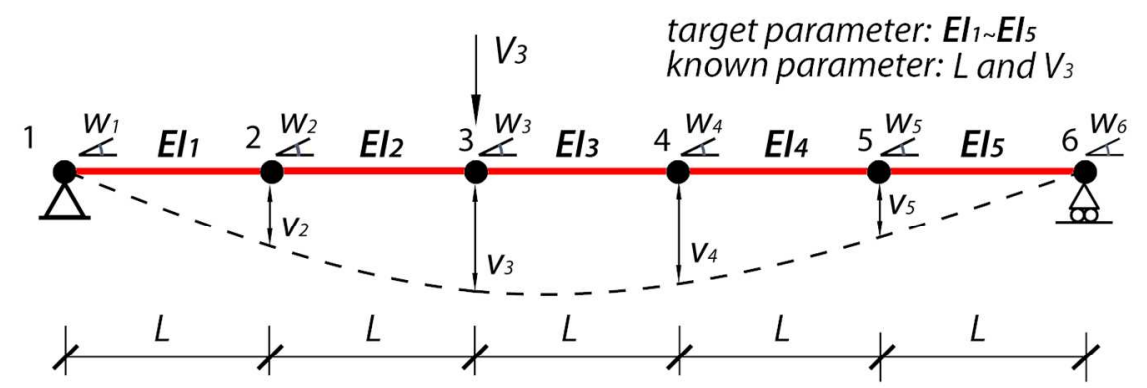

(b)

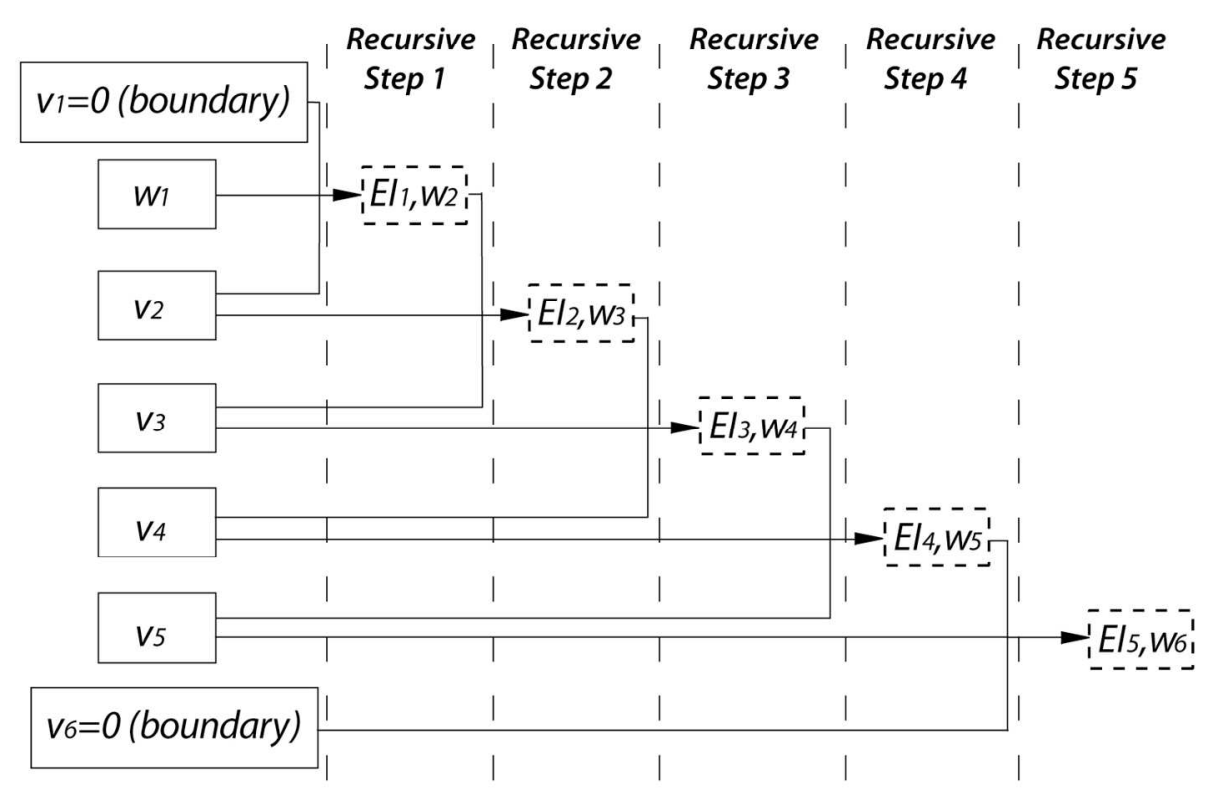

Figure 4. (a) 6-node simply supported beam with 5 target parameters, $\mathrm{EI}_{1} \sim \mathrm{EI}_{5}$, and ten potential measurements, $w_{1} \sim w_{6}$ and $v_{2} \sim v_{5}$; (b) Observability flow for measurement set of $w_{1}, v_{2} \sim v_{5}$

$141 \times 139 \mathrm{~mm}(300 \times 300$ DPI $)$ 


1
2
3
4
5
6
7
8
9
10
11
12
13
14
15
16
17
18
19
20
21
22
23
24
25
26
27
28
29
30
31
32
33
34
35
36
37
38
39
40
41
42
43
44
45
46
47
48
49
50
51
52
53
54
55
56
57
58
60

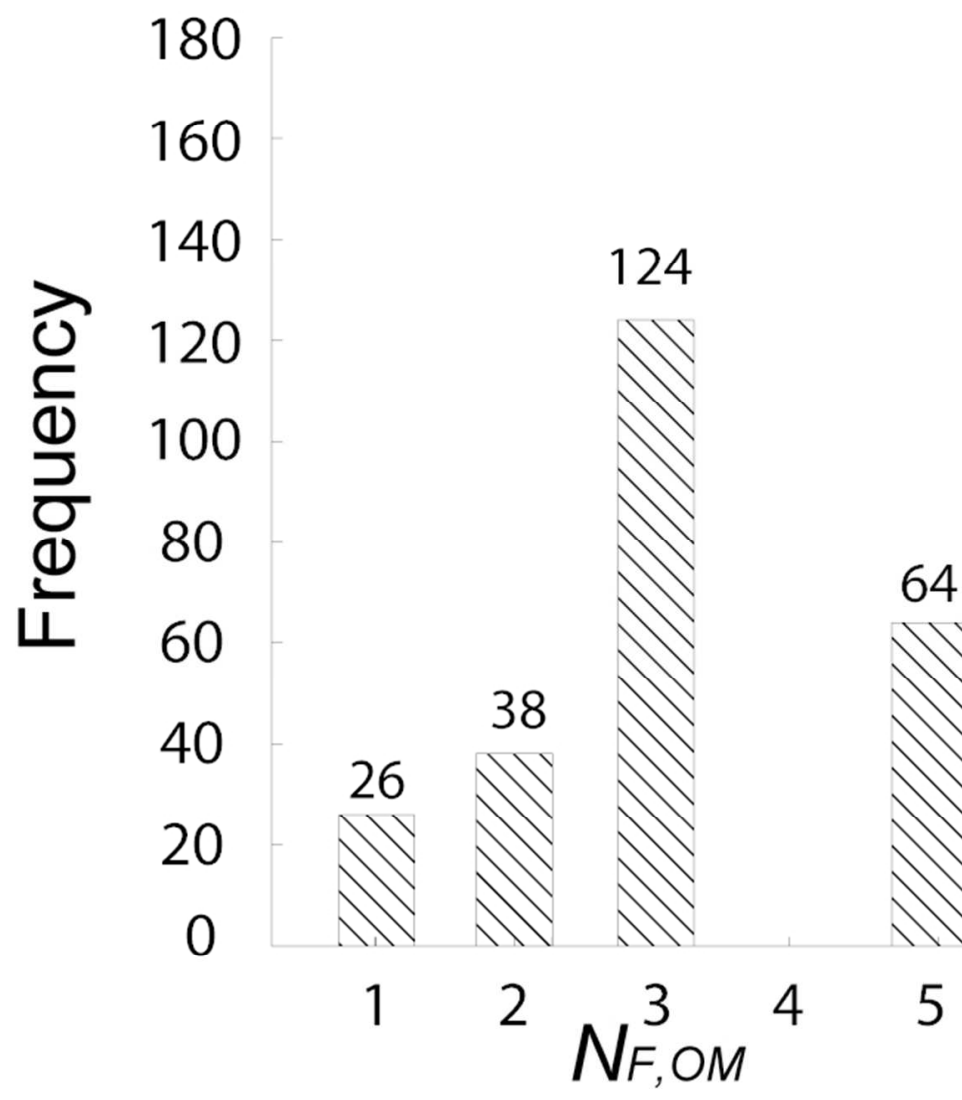

Figure 5. Frequency of the occurrence of the number of identified flexural stiffnesses by $\mathrm{OM}, \mathrm{N}_{\mathrm{F}, \mathrm{OM}}$, equal to 1 to 5 $74 \times 71 \mathrm{~mm}(300 \times 300$ DPI $)$ 


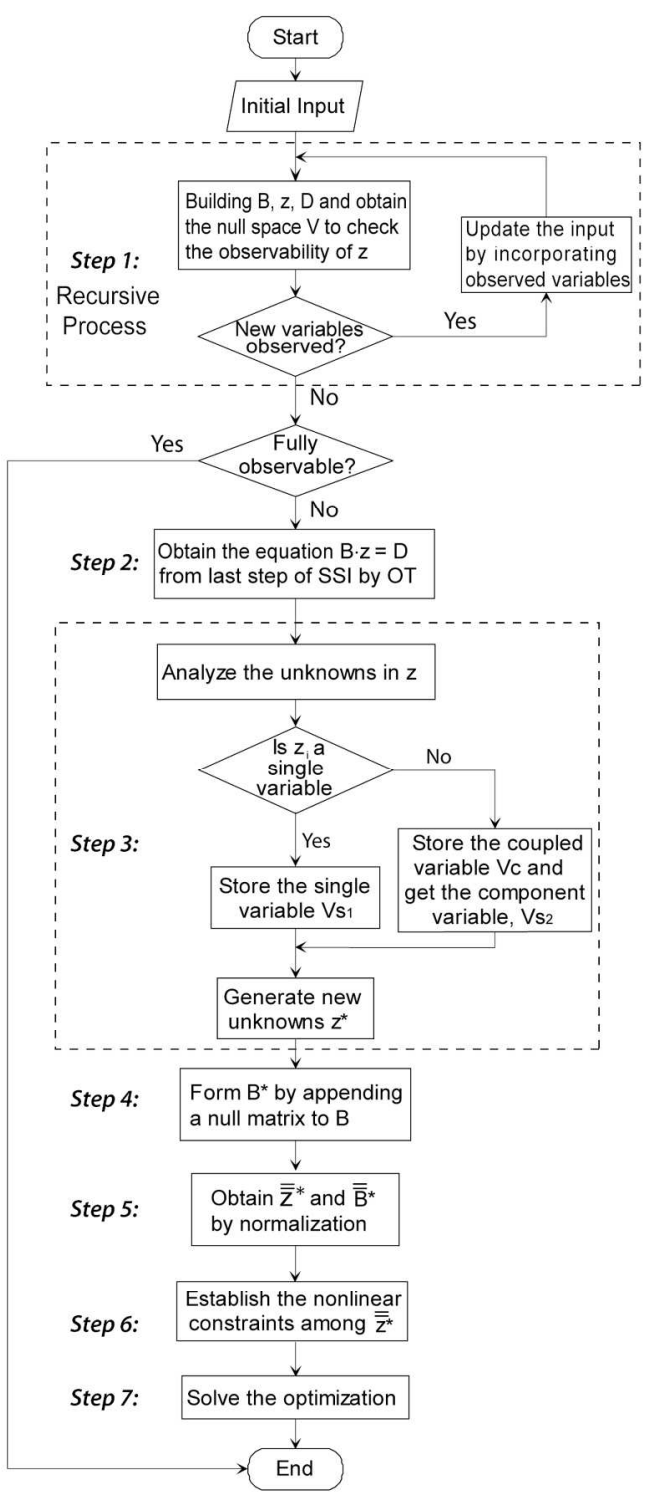

Figure 6. Flow chart of SSI by COM $85 \times 205 \mathrm{~mm}(300 \times 300$ DPI $)$ 
2

3

4

5

6

7

8

9

10

11

12

13

14

15

16

17

18

19

20

21

22

23

24

25

26

27

28

29

30

31

32

33

34

35

36

37

38

39

40

41

42

43

44

45

46

47

48

49

50

51

52

53

54

55

56

57

58

59

60
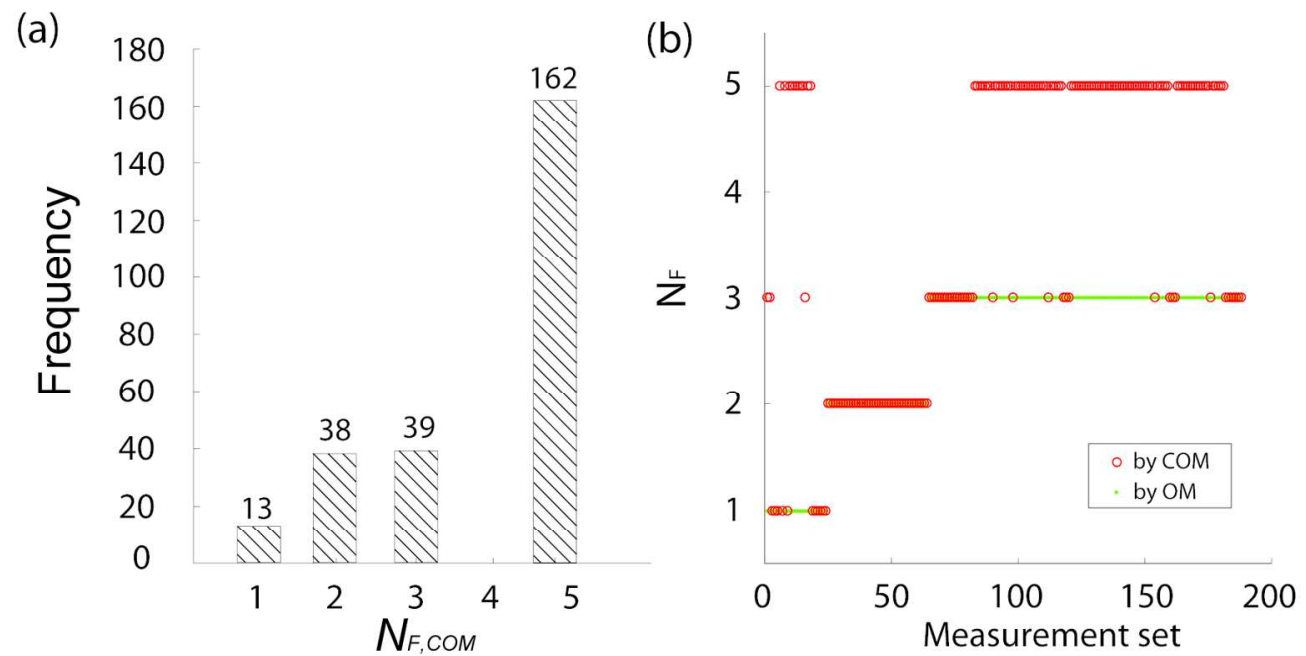

Figure 7. (a) Frequency of the occurrence of the number of identified flexural stiffnesses by $C O M, N_{F, O M}$, equal to 1 to 5 ; (b) Number of observed flexural stiffnesses, $N_{F}$, by OM and COM for the 188 sets with partial observability by OM

$148 \times 77 \mathrm{~mm}(300 \times 300 \mathrm{DPI})$ 


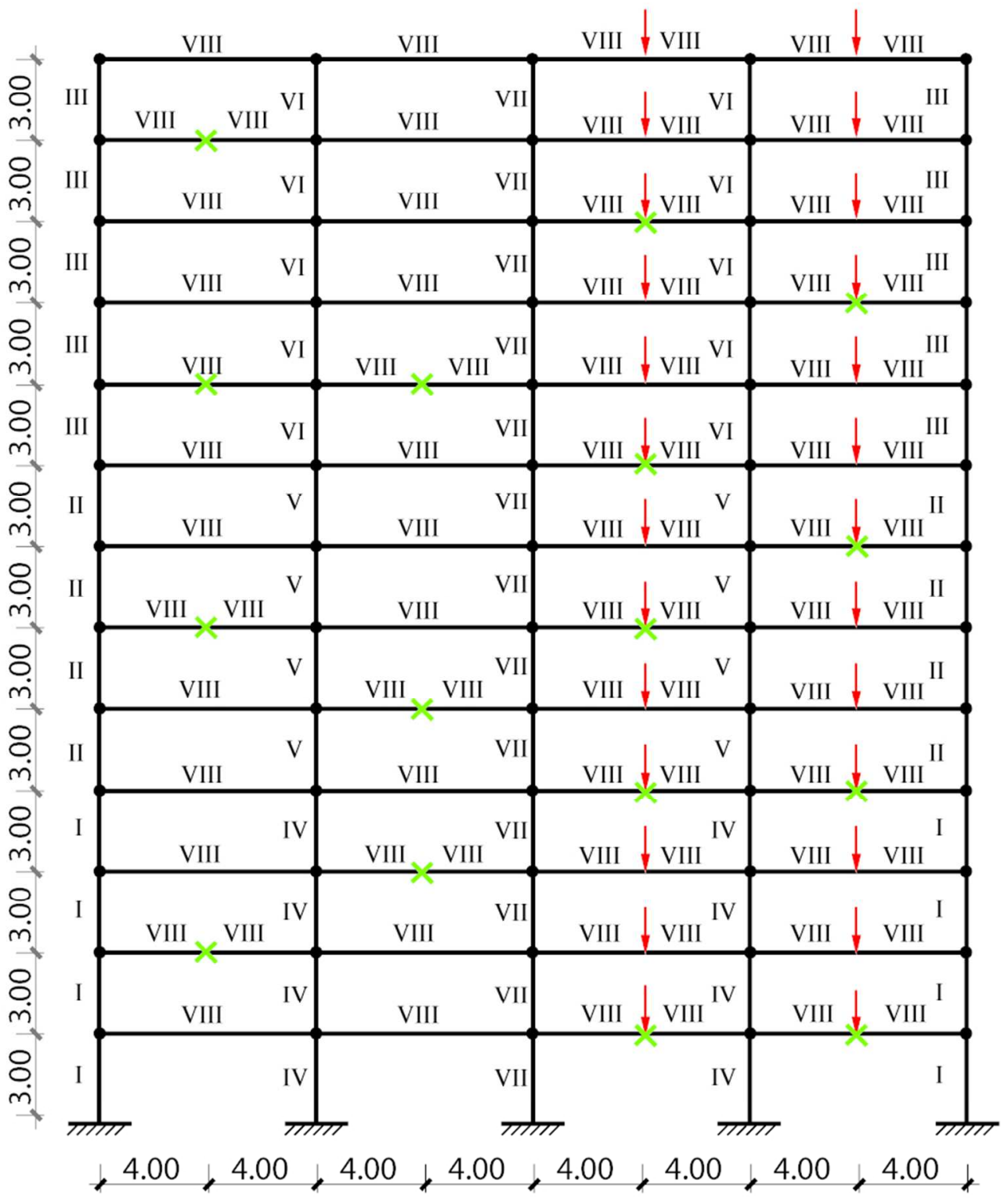

Figure 8. Element type (Roman numerals and see Table 2) of the frame building, the placement of sensors (indicated by cross) and the external load (indicated by arrows)

$82 \times 97 \mathrm{~mm}(300 \times 300 \mathrm{DPI})$ 
(a)

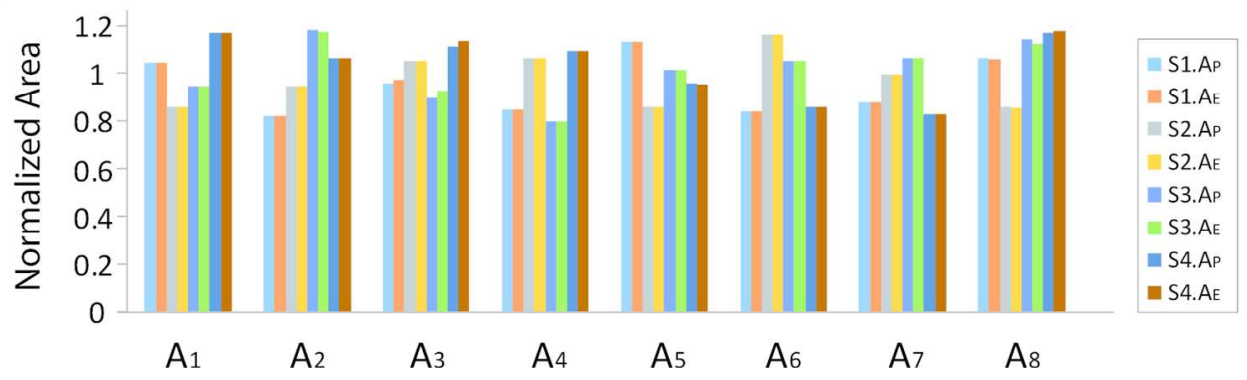

(b)

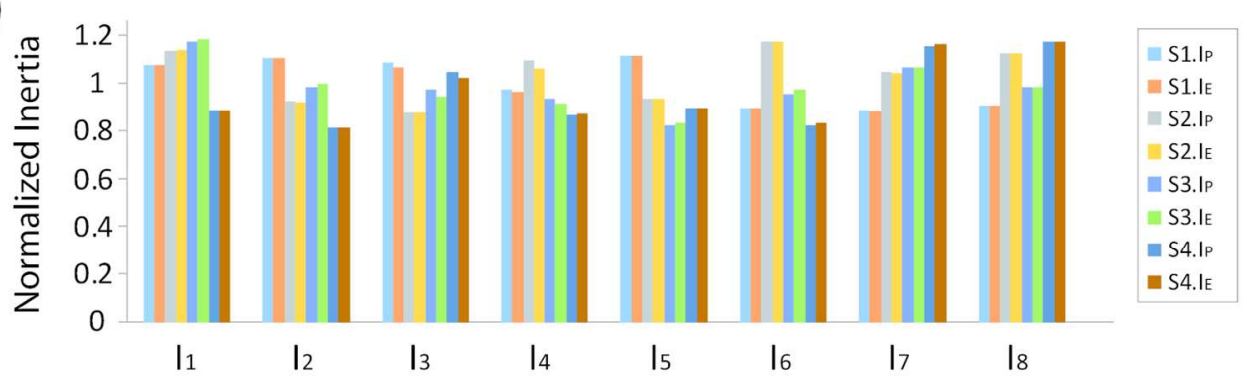

(c)

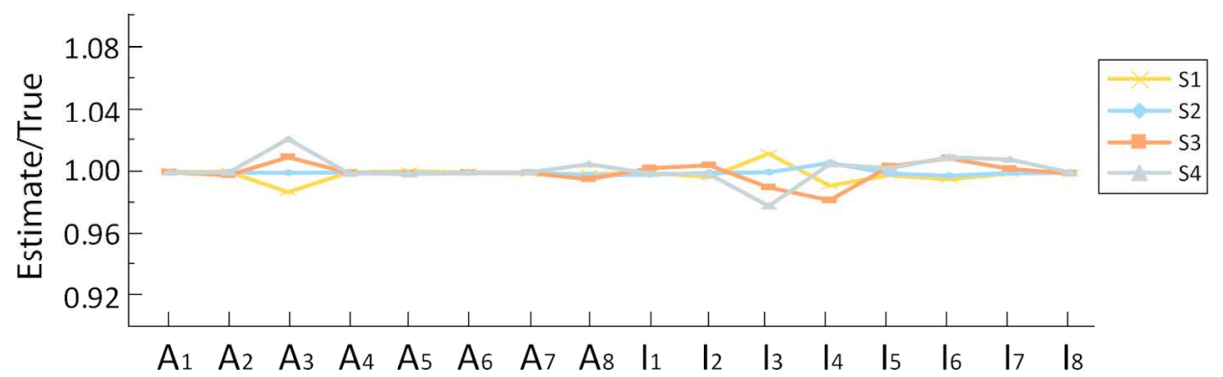

Figure 9. (a) Perturbation factor for set $1 \sim$ set 4, S1.AP S4.AP, and the normalized estimate for set $1 \sim$ set 4 , $S 1 . A_{E} \sim S 4 . A_{E}$, of the eight areas $\left(A_{1} \sim A_{8}\right)$; (b) perturbation factor for set $1 \sim$ set 4 , (S1.IP S4.I $)$ and the normalized estimate (S1. $\left.\mathrm{I}_{\mathrm{E}} \sim \mathrm{S} 4 . \mathrm{I}_{\mathrm{E}}\right)$ of the eight inertias $\left(\mathrm{I}_{1} \sim \mathrm{I}_{8}\right)$; (c) the ratio between the normalized estimates and the perturbation factors for all parameters $\left(A_{1} \sim A_{8}\right.$ and $\left.I_{1} \sim I_{8}\right)$.

$136 \times 129 \mathrm{~mm}(300 \times 300 \mathrm{DPI})$ 